\title{
1 A ROR2 coding variant is associated with craniofacial variation in domestic pigeons
}

3 Authors: Elena F. Boer ${ }^{1}$, Hannah F. Van Hollebeke ${ }^{1}$, Carson Holt ${ }^{2}$, Mark Yandell ${ }^{2}$, and Michael

4 D. Shapiro ${ }^{*}$

5

6 Affiliations: ${ }^{1}$ School of Biological Sciences, University of Utah, Salt Lake City, UT 84112, USA;

$7 \quad{ }^{2}$ Department of Human Genetics and USTAR Center for Genetic Discovery, University of Utah,

$8 \quad$ Salt Lake City, UT 84112, USA

9

10 *Author for correspondence:

11 Michael D. Shapiro, School of Biological Sciences, 257 South 1400 East, Salt Lake City, UT

1284112 USA; phone: +1 801581 5690; fax: +1 801581 4668; email: mike.shapiro@utah.edu 


\section{Summary}

15 Vertebrate craniofacial morphogenesis is a highly orchestrated process that is directed by

16 evolutionarily conserved developmental pathways ${ }^{1,2}$. Within species, canalized developmental

17 programs typically produce only modest morphological variation. However, as a result of

18 millennia of artificial selection, the domestic pigeon (Columba livia) displays radical variation in

19 craniofacial morphology within a single species. One of the most striking cases of pigeon

20 craniofacial variation is the short beak phenotype, which has been selected in numerous

21 breeds. Classical genetic experiments suggest that pigeon beak length is regulated by a small

22 number of genetic factors, one of which is sex-linked (Ku2 locus) ${ }^{3-5}$. However, the molecular

23 genetic underpinnings of pigeon craniofacial variation remain unknown. To determine the

24 genetic basis of the short beak phenotype, we used geometric morphometrics and quantitative

25 trait loci (QTL) mapping on an $F_{2}$ intercross between a short-beaked Old German Owl (OGO)

26 and a medium-beaked Racing Homer $(\mathrm{RH})$. We identified a single locus on the Z-chromosome

27 that explains a majority of the variation in beak morphology in the $\mathrm{RH} \times \mathrm{OGO} \mathrm{F}_{2}$ population. In

differentiated between breeds with short and medium beaks. Within the differentiated Ku2 locus,

we identified an amino acid substitution in the non-canonical Wnt receptor ROR2 as a putative

31 regulator of pigeon beak length. The non-canonical Wnt (planar cell polarity) pathway serves

32 critical roles in vertebrate neural crest cell migration and craniofacial morphogenesis ${ }^{6,7}$. In

33 humans, homozygous ROR2 mutations cause autosomal recessive Robinow syndrome, a rare

34 congenital disorder characterized by skeletal abnormalities, including a widened and shortened

35 facial skeleton ${ }^{8,9}$. Our results illustrate how the extraordinary craniofacial variation among 36 pigeons can reveal genetic regulators of vertebrate craniofacial diversity.

38 Keywords: 
bioRxiv preprint doi: https://doi.org/10.1101/2021.03.15.435542; this version posted March 15, 2021. The copyright holder for this preprint

(which was not certified by peer review) is the author/funder, who has granted bioRxiv a license to display the preprint in perpetuity. It is made available under aCC-BY-NC 4.0 International license.

39 Craniofacial, beak, pigeon, morphometrics, QTL mapping, comparative genomics, non-

40 canonical Wnt signaling pathway, ROR2 


\section{Results and Discussion}

The avian beak shows remarkable diversity among species. Variation in beak

43 morphology within groups like Darwin's finches and Hawaiian honeycreepers illustrates the

44 diversifying potential of natural selection on beak skeletal structures and functions ${ }^{10,11}$. Although

45 the underlying genetic basis of the extraordinary variation among birds remains relatively poorly

46 understood, several genes associated with overall beak size or linear dimensions of beak shape

47 are known in a modest number of species (e.g., COL4A5 in Great tits ${ }^{12}$; IGF1 in Black-bellied

48 seedcrackers ${ }^{13} ; B M P 4, C A L M 1, A L X 1$, and HMGA2 in Darwin's finches $\left.{ }^{14-18}\right)$. Unlike wild birds,

49 the domestic pigeon beak is unconstrained by natural selection; the astounding level of

50 morphological variation within this species is instead the product of intensive artificial selection.

51 Some of the major axes of variation in craniofacial shape that distinguish distantly related avian

52 species are recapitulated among breeds of domestic pigeon, despite different mechanisms of

53 selection between wild and captive populations ${ }^{19,20}$. Therefore, pigeons provide a unique

54 opportunity to uncover genetic variants associated with the types of beak variation that exist

55 throughout the radiation of birds.

57 Pigeon beak length covaries with body size and braincase shape in an experimental cross

To determine the genetic architecture of beak length in pigeons, we established an $F_{2}$

59 intercross between a male Racing Homer $(\mathrm{RH})$ and a female Old German Owl (OGO). The $\mathrm{RH}$,

60 which "has been bred for one purpose - speed - almost to the exclusion of all other factors and

61 traits" ${ }^{21}$, has a medium-length beak that resembles the ancestral condition in rock pigeons

62 (Figure 1A,B). In contrast, the OGO beak "is one of the distinctive characteristics" of the breed

63 and is "short in appearance, which is partly caused by the broad width of the beak in relation to

64 its length" 22 (Figure 1C,D).

65 We scanned the $\mathrm{RH} \times \mathrm{OGO}$ cross founders and $145 \mathrm{~F}_{2}$ individuals using micro-CT, 66 generated 3D surface models of the craniofacial skeleton, and applied a set of 49 landmarks to 
67 the beak and braincase (Supplemental Figure 1, Supplemental Tables 1-2). By calculating the

68 linear distance between the base and tip of the beak and using mass as a proxy for body size ${ }^{23}$,

69 we found a significant positive association between beak length and body size in the $F_{2}$

70 population $\left(R^{2}=0.092, p=0.0001\right.$, Figure $\left.1 E\right)$. We removed the effects of body size variation by

71 fitting a beak length body size linear regression model and found that beak length residuals

72 remained highly variable in the $F_{2}$ population, demonstrating that beak length varies

73 independently of body size (Figure 1F).

74 We also measured three-dimensional (3D) variation in beak and braincase shape

75 through geometric morphometric analysis. In the $\mathrm{RH} \times \mathrm{OGO} \mathrm{F}_{2}$ population, cranium centroid

76 size is negatively associated with body size $\left(R^{2}=0.03, p=0.02\right.$, Supplemental Figure $\left.2 A\right)$. This

77 result contrasts broad patterns of cranium body size allometry observed across diverse

78 pigeon breeds and wild birds ${ }^{20}$, and is likely driven by the exceptionally large cranium and small

79 body size selected for in the OGO breed. In the $F_{2}$ population, cranium centroid size is also

80 negatively associated with curvature from the tip of the beak to the back of the braincase and, to

81 a lesser extent, beak length $\left(R^{2}=0.073, p<0.001\right.$, Supplemental Figure $\left.2 B\right)$. Taken together, the

82 linear and 3D shape analyses reveal subtle but significant relationships between body and

83 cranium size and craniofacial shape in the $\mathrm{RH} \times \mathrm{OGO}$ cross. By shuffling genetic programs for

84 two generations in an experimental cross, we find that body size, cranium size, and beak shape

85 are modular and separable.

86 Although allometry is an important correlate of shape ${ }^{23}$, we focused further analyses on

87 non-allometric craniofacial shape variation. Principal components analysis (PCA) of geometric

88 morphometric shape variables demonstrate that, in the $\mathrm{RH} \times \mathrm{OGO} \mathrm{F}_{2}$ population, the first two

89 principal components (PCs) together account for $50 \%$ of craniofacial shape variation

90 (Supplemental Figure 3A). The principal axis of shape variation (PC1, 39.1\% of shape variation)

91 describes compound variation in beak length and braincase volume (Figure 2A, Supplemental

92 Movie 1) and is strongly correlated with linear measurements of beak length $\left(R^{2}=0.64, p<2.2 e-\right.$ 
93 16, Supplemental Figure 3B). PC2 (10.9\% of shape variation) is defined almost exclusively by

94 changes in braincase shape (Supplemental Figure 3C, Supplemental Movie 2).

Similar to previous findings in domestic pigeons and wild birds ${ }^{20,24}, 3 \mathrm{D}$ beak and braincase shape are strongly integrated in the $\mathrm{RH} \times \mathrm{OGO} \mathrm{F}_{2}$ population ( $r-\mathrm{PLS}=0.923, \mathrm{p}<0.001$,

97 Supplemental Figure $2 \mathrm{~B}$ ). Along the PC1 axis, all $\mathrm{F}_{2}$ individuals are confined to a morphospace

98 defined by the cross founders, but cluster closer to the $\mathrm{RH}$ than the OGO (Figure 2B). This

99 result is reminiscent of our analysis of pigeon beak curvature, in which $\mathrm{F}_{2}$ individuals derived

100 from a straight-beaked Pomeranian Pouter and curved-beaked Scandaroon more closely

101 resembled the Pomeranian Pouter and never achieved the extreme craniofacial curvature of the

102 Scandaroon ${ }^{24}$. Therefore, two different genetic crosses using four different pigeon breeds

103 suggest that the most exaggerated versions of craniofacial traits require coordination of multiple

104 genetic factors.

105

106

Identification of a major-effect beak length QTL on the Z chromosome

107

Next, we used the PC1 scores, which primarily describe variation in beak length, to

110 the $\mathrm{RH} \times \mathrm{OGO}$ cross (log likelihood ratio $(\mathrm{LOD})=23.72$, percent variance explained

$111(\mathrm{PVE})=53.2 \%$, Figure 2C). Nearly identical results were obtained when beak length residuals

112 were used for QTL mapping (Supplemental Figure 4). The identification of a major-effect QTL

113 on the Z-chromosome is consistent with results from classical genetic studies that pointed to a

114 sex-linked regulator of pigeon beak length ${ }^{3-5}$.

115 We next used the peak marker to estimate QTL effects. Within the $F_{2}$ population, male

$116 \mathrm{Z}^{\mathrm{RH}} / \mathrm{Z}^{\mathrm{RH}}$ homozygotes and female $\mathrm{Z}^{\mathrm{RH}} / \mathrm{W}$ hemizygotes had the highest $\mathrm{PC1}$ scores (longest

117 beaks) and were not statistically different from one another (Figure 2D). Male $Z^{\mathrm{RH}} / \mathrm{Z}^{\mathrm{OGO}}$

118 heterozygotes had intermediate PC1 scores (Figure 2D), suggestive of an incompletely 
119 dominant pattern of inheritance. In contrast, female $\mathrm{Z}^{\mathrm{OGO}} \mathrm{W}$ hemizygotes had dramatically lower

120 PC1 scores (shorter beaks) than all other $F_{2}$ individuals carrying the $\mathrm{RH}$ allele (Figure 2D).

121 Although the structure of our experimental cross did not generate homozygous $Z^{\mathrm{OGO}} / \mathrm{Z}^{\mathrm{OGO}}$ males

122 in the $F_{2}$ generation, previous classical genetic studies have demonstrated that, in short-beaked

123 pigeon breeds, body size is sex-associated but beak length is not ${ }^{5,25}$. Therefore, we predict that

124 the beaks of $Z^{\mathrm{OGO}} / \mathrm{Z}^{\mathrm{OGO}}$ males would be indistinguishable from hemizygous $\mathrm{Z}^{\mathrm{OGO}} / \mathrm{W}$ females,

125 although we cannot rule out the possibility that an additional copy of the OGO allele could result

126 in even shorter beaks in $\mathrm{Z}^{\mathrm{OGO}} / \mathrm{Z}^{\mathrm{OGO}}$ males.

127 In summary, our results support the model that pigeon beak length is a polygenic trait

128 controlled largely by one sex-linked factor. Additional minor-effect QTL are likely modifying beak

129 length in the $\mathrm{RH} \times \mathrm{OGO}$ cross, some of which may be detectable in a larger $\mathrm{F}_{2}$ population or an

$130 \quad \mathrm{~F}_{3}$ generation that includes $\mathrm{Z}^{\mathrm{OGO}} / \mathrm{Z}^{\mathrm{OGO}}$ males.

A ROR2 coding variant is associated with beak length across diverse domestic pigeon breeds

The beak length QTL represents a relatively large (3.6-Mb) genomic region that includes

134 several genes expressed during pigeon craniofacial development (Supplemental Figure 5,

135 Supplemental Table 3), thus limiting our ability to pinpoint the specific gene(s) and mutation(s)

136 that regulate beak length in the $\mathrm{RH} \times \mathrm{OGO}$ cross. In addition, because the mapping population

137 was derived from just two birds that represent a fraction of the morphological diversity across

138 pigeon breeds, we have no way of knowing if the beak length QTL we identified is relevant

139 beyond the $\mathrm{RH} \times \mathrm{OGO}$ cross. Short beaks are characteristic of numerous related pigeon breeds

140 that belong to the Owl family, but have also been selected for in a variety of unrelated non-Owl

141 breeds ${ }^{26-28}$. Pigeon breeders might have repeatedly selected the same standing variant in

142 different breeds, independent variants of the same gene in different breeds, or different genes

143 altogether in different breeds. 
To distinguish between independent and shared genetic origins of short beaks, we scanned for genomic variants associated with beak length across diverse pigeon breeds by comparing resequenced genomes of 56 short-beaked individuals from 31 (7 Owl and 24 non-

147 Owl) breeds to 121 genomes from 58 medium- or long-beaked breeds and feral pigeons (Figure

$1483 \mathrm{~A}-\mathrm{B})$. We then searched for genomic regions that were differentiated between these groups 149 using two related differentiation statistics $\left(\mathrm{wcF}_{\mathrm{ST}}{ }^{29}\right.$ and $\left.\mathrm{pF}_{\mathrm{ST}}{ }^{30}\right)$. A $\sim 293-\mathrm{kb}$ segment on the Z150 chromosome scaffold ScoHet5_445.1 stood out as significantly differentiated between the short151 and medium/long-beaked groups (top $0.1 \%$ by wcF $\mathrm{ST}_{\mathrm{ST}}$; Figure 3C-D, Supplemental Figure 6) and 152 was located within the genomic interval identified in our QTL scan. In the peak differentiated 153 region, short-beaked pigeons displayed elevated levels of haplotype homozygosity relative to 154 medium/long-beaked individuals, providing further support for widespread positive selection on 155 this locus in short-beaked breeds (Figure 3E). Thus, the short beak allele identified in our QTL mapping experiments is not specific to either the OGO cross founder or the Owl family. Instead,

157 the short beak allele on the Z-chromosome likely arose once and was repeatedly selected in 158 different breeds.

The single most significantly-differentiated SNP genome wide $\left(\mathrm{wcF}_{\mathrm{ST}}=0.88, \mathrm{pF}_{\mathrm{ST}}=0\right)$ is 160 located at scaffold position ScoHet5_445.1:6568443. The non-reference allele causes a 161 missense substitution in the seventh exon of $R O R 2\left(R O R 2^{C 1087 T}\right.$, hereafter the Ku2 allele $\left.{ }^{5}\right)$ in 162 short-beaked pigeons. ROR2 encodes a noncanonical Wnt receptor with well-established roles 163 in cell polarity and motility in multiple embryonic tissues, including the neural crest ${ }^{31}$. This gene

164 is required for normal craniofacial development: in humans, homozygous mutations in ROR2 165 cause autosomal recessive Robinow syndrome, a severe skeletal dysplasia characterized by 166 extensive abnormalities, including a prominent forehead (frontal bossing), wideset eyes 167 (hypertelorism), and a broad, short nose ${ }^{8,9}$. In mice, Ror2 knockout or knock-in of Robinow168 associated mutations disrupts endochondral bone development and causes profound skeletal 169 abnormalities, including craniofacial outgrowth defects ${ }^{32-34}$. Likewise, the OGO and 
170 morphologically similar pigeon breeds have reduced craniofacial outgrowths in the form of short

171 beaks.

172 Within the short-beaked group, $98 \%$ of pigeons (45/46) were homozygous or

173 hemizygous for the Ku2 allele; only the Chinese Nasal Tuft, a breed that can have a short- or

174 medium-length beak ${ }^{35}$, was heterogyzous. In contrast, 93\% (97/104) of medium-beaked birds

175 were homozygous, hemizygous, or heterozygous for the ancestral allele (Figure 3F). A genome-

176 wide scan for putatively damaging coding variants predicted that the Ku2 allele is both highly

177 deleterious and associated with short beaks (VAAST ${ }^{36}$ top-ranked feature, score=64.37, $\mathrm{p}=4 \mathrm{e}$ -

178 8). At the amino acid level, the Ku2 allele causes an arginine-to-cysteine transition in the ROR2

179 extracellular kringle fold, a cysteine-rich, disulfide-bonded domain that is unlikely to tolerate

180 mutations due to its small size and complex folding ${ }^{37}$. The precise number and spacing of

181 cysteine residues in the kringle domain are deeply conserved in vertebrate ROR2 and

182 invertebrate Ror homologs (Figure 3G), suggesting that the ectopic cysteine residue introduced

183 by the Ku2 mutation may have a substantial impact on disulfide bond formation and kringle

184 domain folding. Although the precise function of the ROR2 kringle domain remains unclear, it is

185 thought to mediate protein-protein interactions and may modulate the affinity of the adjacent

186 Frizzled-like ligand-binding domain for WNT5A ${ }^{38,39}$.

187 Like the pigeon Ku2 allele, the majority of known Robinow-associated missense

188 mutations in human patients are clustered in the kringle and Frizzled-like extracellular domains.

189 All of the characterized disease variants cause increased ROR2 protein retention in the

190 endoplasmic reticulum, suggesting that the extracellular domain must be properly folded before

191 transport to the plasma membrane ${ }^{37,40}$. Based on available evidence, we hypothesize that the

192 Ku2 allele disrupts ROR2 protein folding in short-beaked pigeons, resulting in craniofacial

193 outgrowth anomalies similar to Robinow syndrome in humans.

195 ROR2 and WNT5A are expressed during pigeon craniofacial morphogenesis 
In chicken and mouse embryos, ROR2 expression is widespread with regions of strong expression in the facial prominences, dorsal root ganglia, and limb buds ${ }^{31,41,42}$. Using RNA-seq,

198 we found that both ROR2 and WNT5A are also strongly expressed in short- and medium-

199 beaked pigeon facial primordia ( $n=5$ each), with higher expression in the frontonasal and 200 maxillary prominences (upper beak) relative to the mandibular prominence (lower beak; Figure $2014 \mathrm{~A}-\mathrm{B})$. Neither ROR2 nor WNT5A is differentially expressed between short- and medium202 beaked embryos at the pigeon equivalent of chicken stage HH29 (Ref. 43, Figure 4A-B), an 203 early embryonic stage at which distinct craniofacial morphologies are evident among avian 204 species $^{44}$.

205 Spatial expression of ROR2 is broad during early pigeon facial development (HH21-29), 206 with higher levels in the FNP and lateral nasal prominences (LNP) at HH25 and HH29 (Figure 207 4C-E). WNT5A expression domains overlap with ROR2, but are more spatially restricted to the 208 regions of the facial primordia that will grow out to form the beak (Figure 4F-H), similar to mouse 209 and chicken ${ }^{45,46}$. Thus, ROR2 and WNT5A are expressed together in pigeons in a spatial and 210 temporal manner that is consistent with their role as regulators of craniofacial morphogenesis.

211 The lack of differential ROR2 expression in short- and medium-beaked pigeon embryos 212 implicates the Ku2 coding mutation, rather than differences in the regulation of expression, in

213 the development of the short beak phenotype.

Pigeons model vertebrate evolution and disease

Several developmental pathways have been implicated in the evolution of beak diversity

217 in other birds, including Darwin's finches, Great tits, and Black-bellied seedcrackers ${ }^{12-16}$, 218 Although ROR2 has a well-established role in mammalian craniofacial development, to our 219 knowledge, this is the first example of a member of the noncanonical Wnt signaling pathway 220 regulating craniofacial development and diversity in birds. This finding contrasts with other 221 examples of recurrent evolution of derived traits via changes in the same genes in pigeons and 
222 other species, including head crests (EPHB2, also in ringneck doves ${ }^{27,47}$ ), feathered feet

$223\left(P I T X 1\right.$ and TBX5, also in chickens $\left.{ }^{30,48}\right)$, and plumage color patterning (NDP, also in crows ${ }^{49-}$

$\left.22{ }^{51}\right)$. Considering the deep evolutionary conservation of developmental pathways that regulate

225 craniofacial morphogenesis, our findings raise the possibility that noncanonical Wnt signaling is

226 modulated in other cases of avian craniofacial variation. We did not identify noncanonical Wnt

227 pathway genes in our recent study of the genetic basis of pigeon beak elaboration ${ }^{24}$,

228 suggesting that distinct genetic programs underlie reduction and exaggeration of the same

229 tissues and structures.

230 The identification of ROR2 as a putative regulator of beak length adds to a growing list of

231 genes that underlie morphological variation in the domestic pigeon and are associated with

232 human diseases, including congenital defects and cancer ${ }^{30,50,52,53}$. In addition, prior work in

233 pigeons has predicted the molecular basis of diverse morphological traits in other wild and

234 domestic species ${ }^{27,30,47,49-51}$. Thus, the pigeon is an exceptional model to interrogate the genetic

235 underpinnings of vertebrate evolution, development, and disease.

\section{Acknowledgments}

238 We are grateful to Nathan Young and Rich Schneider for generously sharing their time and 239 expertise related to geometric morphometrics, craniofacial biology, and avian embryology. We

240 thank all past and present members of the Shapiro Lab, including Rebecca Bruders, Alexa

241 Davis, Emily Maclary, Anna Vickrey, and Ryan Wauer for help with animal husbandry, technical

242 assistance, and advice. We are grateful to Emily Maclary for comments on the manuscript. We

243 thank members of the Utah Pigeon Club and National Pigeon Association for sample

244 contributions. We acknowledge the University of Utah Preclinical Imaging Core Facility,

245 especially Tyler Thompson, for micro-CT imaging; the Center for High Performance Computing

246 at the University of Utah for computing resources; the University of Utah High-Throughput 
247 Genomics Shared Resource for RNA library preparation and sequencing; and the University of

248 Minnesota Genomics Core for GBS library preparation and sequencing.

\section{Author Contributions}

251 Conceptualization, E.F.B. and M.D.S.; Methodology, E.F.B. and M.D.S.; Software, C.H. and

252 M.Y; Investigation, E.F.B. and H.F.V.; Resources, C.H. and M.Y; Formal Analysis, E.F.B.;

253 Writing - Original Draft, E.F.B. and M.D.S.; Writing - Review \& Editing, E.F.B., H.F.V., and

254 M.D.S.; Funding Acquisition, E.F.B. and M.D.S.; Visualization, E.F.B.; Supervision, M.D.S.

\section{Declaration of Interests}

257 The authors declare no competing interests.

\section{Figure Legends}

260 Figure 1. Beak length variation in a pigeon $\mathbf{F}_{2}$ intercross. (A) Representative image of the 261 medium beak Racing Homer $(\mathrm{RH})$ breed. (B) 3D surface model of the craniofacial skeleton of 262 the male $\mathrm{RH}$ founder. (C) Representative image of the short beak Old German Owl (OGO) 263 breed. (D) Surface model of the craniofacial skeleton of the female OGO founder. (E) Raw beak 264 length (measured in arbitrary units) vs. total body mass (measured in grams) in the $\mathrm{RH} \times \mathrm{OGO}$ 265 cross. Gray line indicates linear model from beak length mass regression. (F) Distribution of 266 residuals from beak length $\sim$ mass regression. For $(E-F)$, black dots denote $\mathrm{RH} \times \mathrm{OGO} \mathrm{F}_{2}$ 267 individuals, red triangle is OGO founder, blue square is $\mathrm{RH}$ founder. Image credits: Sydney 268 Stringham (A), Brian McCormick (B).

270 Figure 2. A major-effect QTL on the Z-chromosome is associated with principal

271 component 1 (PC1) in the $\mathrm{RH} \times$ OGO F2 population. (A) Visualizations of geometric 272 morphometric PC1 minimum and maximum shapes three ways: warped 3D surface meshes 
273 (left), wireframes showing displacement of landmarks from mean shape (center), and heatmaps

274 displaying regional shape variation (right). For warped meshes and wireframes, shape changes

275 are magnified 1.5x to aid visualization. (B) PCA plot of PC1 vs. PC2. (C) Genome-wide QTL

276 scan for PC1 reveals significant QTL on Z linkage group. (D) PC1 effect plot estimated from

277 QTL peak marker. Letters denote significance groups; $\mathrm{RH}=$ allele from $\mathrm{RH}$ founder, $\mathrm{OGO}=$

278 allele from OGO founder.

Figure 3. Comparison of short beak and medium/long beak pigeon genomes reveals

281 ROR2 coding variant. (A-B) Representative images of individuals representing short beak (A) 282 and medium or long beak (B) pigeon breeds. (A) Short beak pigeons, from left to right: English 283 Short Face Tumbler, African Owl, Oriental Frill, Budapest Tumbler. (B) Medium/long beak 284 pigeons, from left to right: West of England, Cauchois, Scandaroon, Show King. (C) Genomewide scan for allele frequency differentiation between short beak $(n=56)$ and medium/long beak $(n=121)$ pigeons. (D) Region of peak $F_{S T}$ on ScoHet5_445.1; black horizontal bars represent four genes in the region. For (C-D), genomic scaffolds are colored in gray and ordered by genetic position in $\mathrm{RH} \times \mathrm{OGO}$ linkage map; black dots indicate SNPs that are significantly differentiated by $\mathrm{pF}_{\mathrm{ST}}$ (Bonferroni-corrected $\mathrm{p}$-value $<0.05$ ); red dots are significant SNPS located on scaffold ScoHet5_445.1; dashed horizontal line represents threshold for genome-

291 wide top $0.1 \%$ of differentiated SNPs by wcF ST; $_{\text {; }}$ arrow points to ScoHet5_445.1:6568443, the 292 most differentiated SNP ( $\left.F_{S T}=0.88\right)$ genome-wide. $(E)$ Extended haplotype homozygosity in $F_{S T}$ 293 peak region; dotted vertical line indicates position of ScoHet5_445.1:6568443; smoothed lines 294 represent local regression fitting ${ }^{54}$. (F) Histogram of genotypes at ScoHet5_445.1:6568443 in 295 short beak and medium/long beak groups. (G) Amino acid alignment of kringle domain from 296 vertebrate ROR2 and invertebrate Ror homologs. The Ku2 allele causes an arginine-to-cysteine 297 substitution in short beak pigeon breeds. 
Figure 4. ROR2 and WNT5A are expressed in pigeon facial primordia. (A-B) $R O R 2(\mathrm{~A})$ and WNT5A (B) mRNA expression in facial primordia that will form the upper and lower beak from HH29 short and medium beak pigeon embryos. Each individual embryo is displayed in a different color. $(\mathrm{C}-\mathrm{H})$ Whole-mount in situ hybridization for ROR2 (C-E) and WNT5A (F-H) in medium beak pigeon embryos at $\mathrm{HH} 21, \mathrm{HH} 25$, and $\mathrm{HH} 29$. ROR2 is broadly expressed in facial primordia at all stages. WNT5A is strongly expressed in the FNP and the lateral edges of the MXP and LNP, with increased expression at the edge of the MDP at HH29. Letters indicate embryonic tissues/structures: $\mathrm{e}=\mathrm{eye}, \mathrm{fnp}=$ frontonasal prominence, I=lateral nasal prominence, $\mathrm{mdp}=$ mandibular prominence, $\mathrm{mxp}=$ maxillary prominence.

Supplemental Figure 1. Pigeon craniofacial landmark atlas. Landmarks are indicated by blue discs.

Supplemental Figure 2. Allometry and integration in the RH x OGO cross. (A) Cranium centroid size $\sim$ body size (mass) linear regression. (B) Cranium shape $~$ centroid size linear regression. (B) Beak vs. braincase PLS1 shapes. Minimum and maximum shapes are depicted as wireframes and/or warped meshes along corresponding axis.

$320 \mathrm{RH} \times \mathrm{OGO} \mathrm{F}_{2}$ individuals. (C) Minimum and maximum PC2 shapes depicted as warped mesh

321 (left), wireframe showing landmark displacement (center), or heatmap indicating regional shape 322 changes (right). For mesh and wireframe models, shape change is magnified $1.5 \mathrm{x}$ to aid 323 visualization. 
Supplemental Figure 4. QTL scan using beak length residuals. (A) Genome-wide QTL scan for beak length using residuals from beak length body mass linear regression. (B) LOD

327 support interval is nearly identical to PC1 QTL interval (displayed in Figure 2D). Genes in

328 interval on ScoHet5_445.1 are displayed at bottom and color-coded by expression level. (C)

329 Plot of QTL effects, using peak marker highlighted by black dot in (B).

Supplemental Figure 5. PC1 QTL support interval. (A) LOD support interval for QTL on Z.

332 Markers in interval are denoted with dots and labeled with genomic scaffold name

333 (ScoHet5_445.1 or ScoHet5_227) and position (in Mb); black dot indicates QTL peak marker

334 (ScoHet5_445.1:6.57-Mb), which was used to estimate QTL effects. (B) Genes in QTL interval, 335 color-coded by mRNA expression level in facial primordia derived from $\mathrm{HH} 29 \mathrm{RH}$ embryos. $336 R O R 2$ is located directly under QTL peak.

Supplemental Figure 6. Allele frequency differentiation $\left(F_{S T}\right)$ and extended haplotype homozygosity (EHH) on scaffold ScoHet5_445.1 in short and medium/long beak pigeons.

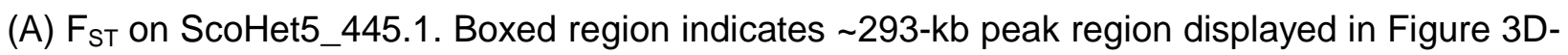

342 line indicates position of ScoHet5_445.1:6568443.

344 Supplemental Movie 1. PC1 shape variation. Minimum to maximum, magnified 1.5x.

345 Supplemental Movie 2. PC2 shape variation. Minimum to maximum, magnified 1.5x.

346 Supplemental Table 1. Description of skull and jaw landmarks.

347 Supplemental Table 2. Landmark pairs used for skull and jaw linear measurements.

348 Supplemental Table 3. Genes in the PC1 QTL interval. 


\section{Resource Availability}

352 Lead Contact

353 Further information and requests for resources and reagents should be directed to and will be

354 fulfilled by the Lead Contact, Michael Shapiro (mike.shapiro@utah.edu).

Materials Availability

357 Plasmids generated to synthesize RNA in situ hybridization probes against pigeon $R O R 2$ and

358 WNT5A are available upon request.

Data and Code Availability

361 Whole genome sequencing and RNA-sequencing datasets generated for this study have been

362 deposited to the NCBI SRA database under BioProject PRJNA680754. Additional short-beaked

363 genomes are available under BioProject PRJNA513877 (SRR8420387-SRR8420391,

364 SRR8420393, SRR8420394, SRR8420397).

\section{Experimental Model and Subject Details}

367 Columba livia

368 Pigeons were utilized in accordance with protocols approved by the University of Utah 369 Institutional Animal Care and Use Committee (protocols 10-05007, 13-04012, and 19370 02011). Further information is provided throughout the Method Details section.

\section{Method Details}

$373 \mathrm{RH} \times \mathrm{OGO} \mathrm{F}_{2}$ intercross and $3 \mathrm{D}$ imaging

$374 \quad \mathrm{~A} \mathrm{~F}_{2}$ intercross was established between a male Racing Homer $(\mathrm{RH})$ and a female Old German

375 Owl (OGO). $F_{1}$ hybrids $(n=15)$ were interbred to generate a $F_{2}$ mapping population. $F_{2}$ offspring 376 that reached 6 months of age $(n=145)$ were euthanized and basic biometrics (e.g. mass) were 
recorded. All $F_{2}$ offspring and cross founders were submitted to the University of Utah

378 Preclinical Imaging Core Facility for micro-CT imaging. For each bird, a whole-body scan was

379 performed on a Siemens Inveon micro-CT using the following parameters: voxel size=94 $\mu$,

380 photon voltage $=80 \mathrm{kV}$, source current $=500 \mu \mathrm{A}$, exposure time $=200 \mathrm{~ms}$. Scans were

381 reconstructed using a Feldkamp algorithm with Sheep-Logan filter and a calibrated beam

382 hardening correction.

\section{Surface model generation and landmarking}

385 Surface model generation and landmarking were performed as described ${ }^{24}$. Briefly, a substack 386 containing the cranium was extracted from the whole-body DICOM file stack in ImageJ v1.52q,

387 exported as a NifTI file ( ${ }^{*}$.nii), and imported into Amira v6.5.0 (Thermo Fisher Scientific). Using 388 the Segmentation Editor threshold feature, the cranial skeleton was segmented from soft tissue 389 and exported as a HxSurface binary ( ${ }^{*}$.surf) file. Surface meshes were converted to Polygon 390 (Stanford) ASCII ( ${ }^{\star}$.ply) files using i3D Converter v3.80 and imported into IDAV Landmark Editor 391 v3.0 (UC Davis) for landmarking. We applied a set of landmarks (Supplemental Figure 1, 392 Supplemental Tables 1-2) to the braincase ( $n=29$ landmarks) and upper beak $(n=20)$ of all $F_{2}$ 393 individuals and the cross founders. Landmark coordinates were exported as a NTsys landmark 394 point dataset ( ${ }^{*}$.dta) for geometric morphometric analysis.

396 Blood collection and genomic DNA extraction

397 Blood samples from adult pigeons used for whole-genome sequencing were collected at local 398 pigeon shows, at breeder's homes, or in the Shapiro lab loft. $\mathrm{RH} \times \mathrm{OGO} \mathrm{F}_{2}$ offspring were bled 399 at time of fledging (approximately 1 month of age). For each individual, a blood sample was 400 collected from the brachial vein and stored in an EDTA-coated sample tube at $-80^{\circ} \mathrm{C}$. RNase- 
401 treated genomic DNA was extracted using a DNeasy Blood and Tissue Kit and eluted in Buffer

402 EB (Qiagen).

403

404

Genotyping-by-sequencing (GBS) and linkage map assembly

405 Genomic DNA samples from $\mathrm{RH} \times \mathrm{OGO}$ cross founders and $171 \mathrm{~F}_{2}$ offspring were submitted to

406 the University of Minnesota Genomics Core for GBS library prep and sequencing. Genomic

407 DNA samples were digested with ApeKI (NEB), then ligated with T4 ligase (NEB) and phased

408 adaptors with CWG overhangs. The ligated samples were purified with SPRI beads and

409 amplified for 18 PCR cycles with 2X NEB Taq Master Mix to add barcodes. Libraries were

410 purified, quantified, pooled, size selected for the 624-724 bp library region (480-580 DNA

411 insert), and treated with ExoVII to remove any remaining single stranded material. The final pool

412 was diluted to $1 \mathrm{nM}$ for sequencing on the Illumina NovaSeq 6000 using single-end 1 X100

413 reads. Target sequencing volume was $\sim 4.75 \mathrm{M}$ reads/sample. Sequencing read quality was

414 assessed with FastQC (Babraham Bioinformatics) and Illumina adapters were trimmed with

415 Cutadapt ${ }^{55}$. Reads were mapped to the Cliv_2.1 reference assembly ${ }^{56}$ using Bowtie $2{ }^{57}$.

416 Genotypes were called using the Stacks v2.52 ref_map.pl program, which executes the Stacks

417 pipeline programs gstacks and populations. The following options were passed to populations: -

418 H -r 0.75 --map-type F2 --map-format rqtl.

419 The $\mathrm{RH} \times$ OGO genetic map was constructed with the $\mathrm{R}$ package R/qtl v1.46-2 using

420 genotype data from $171 \mathrm{~F}_{2}$ individuals. Because of differences in segregation patterns,

421 autosomal and Z-linked scaffolds were assembled separately. For autosomal scaffolds, markers

422 with identical genotypes or displaying segregation distortion (chi-square $p<0.005$ ) were

423 eliminated. Preliminary filtering was performed to remove markers missing in more than $20 \%$

$424(34 / 170)$ of $F_{2}$ individuals. Pairwise recombination fractions were calculated and a preliminary

425 genetic map was estimated using the est.rf and est.map functions, respectively. The

426 droponemarker and calc.errorlod functions were used with the parameter (error.prob $=0.005$ ) to 
427 identify problematic markers and likely genotyping errors, which were eliminated from the

428 genetic map. Linkage groups were formed using the function formLinkageGroups with

429 parameters (max.rf $=0.25$, min.lod $=6$ ). For the Z-chromosome, the same workflow was carried

430 out, except that distorted markers were not removed. Preliminary marker ordering was done for

431 all linkage groups using the orderMarkers function with the parameter (window size $=7$ ). Final

432 marker ordering was completed manually based on calculated recombination fractions and LOD

433 scores. The compareorder function was used to test alternative marker orders; changes in

434 marker ordering that resulted in an increased LOD score and decreased linkage group length

435 were retained. The final $\mathrm{RH} \times$ OGO genetic map is composed of 6128 markers (5553

436 autosomal, 575 Z-linked) on 35 linkage groups (34 autosomal, 1 Z-linked) with a genotyping rate

437 of $90.1 \%$.

Whole-genome resequencing

440 For the current study, we resequenced genomes for 33 pigeons from 24 short-beaked breeds:

441 African Owl, Australian Tumbler, Berlin Short Face Tumbler, Budapest Tumbler, Canario

442 Cropper, Chinese Nasal Tuft, Classic Old Frill, Damascene, Egyptian Swift, English Long Face

443 Tumbler, English Short Face Tumbler, Granadino Pouter, Hamburg Sticken, Helmet, Italian Owl,

444 Long Face Muff Tumbler, Nun, Old German Owl, Oriental Frill, Rafeno Pouter, Russian

445 Tumbler, Taganrog Tumbler, Temeschburger Schecker, Uzbek Tumbler. We also resequenced

44629 pigeons from 24 medium- or long-beaked breeds: Berlin Long Faced Tumbler, Dragoon,

447 English Carrier, English Magpie, Scandaroon, Racing Homer, Danzig Highflier, Schalkaldener

448 Mohrenkopf, Fairy Swallow, Hungarian Giant House Pigeon, Crested Saxon Field Color Pigeon,

449 Saint, Franconian Trumpeter, American Highflier, Bokhara Trumpeter, Komorner Tumbler,

450 Brunner Pouter, Mindian Fantail, Naked Neck, Turkish Tumbler, Norwich Cropper, Miniature

451 American Crest, Rhine Ringbeater, Vienna MF Tumbler. 
Genomic DNA samples were submitted to the High-Throughput Genomics and

453 Bioinformatic Analysis Shared Resource at the University of Utah for library preparation and

454 sequencing. DNA libraries were prepared using the Illumina TruSeq DNA PCR-Free Sample

455 Preparation Kit with an average insert size of 350 bp. 125-cycle paired-end sequencing was

456 performed on an Illumina HiSeq 2500 instrument (3-4 libraries/lane).

458 Embryonic tissue isolation and RNA extraction

459 Pigeon eggs were collected from Racing Homer (medium beak) and Oriental Frill (short beak)

460 breeding pairs and incubated to embryonic day 6 (Hamburger-Hamilton (HH) stage $28-29,{ }^{58}$ ).

461 Facial prominences that form the upper beak (frontonasal and maxillary, FNP+MXP) and lower

462 beak (mandibular, MDP) were dissected and stored separately in RNAlater (ThermoFisher

463 Scientific) at $-80^{\circ} \mathrm{C}$. Additional tissue was harvested from each embryo and used for DNA

464 extraction and sex determination following a previously published PCR-based assay ${ }^{59}$. Total

465 RNA was extracted from embryonic tissue samples using the RNeasy Mini Kit with RNase-Free

466 DNAse Set and a TissueLyser LT (Qiagen).

RNA-sequencing

469 Total RNA from FNP+MXP and MDP samples from HH28-29 female Racing Homer $(n=5)$ and

470 Oriental Frill $(n=5)$ embryos was submitted to the High-Throughput Genomics and Bioinformatic

471 Analysis Shared Resource at the University of Utah for library preparation and sequencing. RNA

472 sample quality was assessed using the RNA ScreenTape Assay (Agilent). For each sample, a

473 stranded sequencing library was prepared using the TruSeq Stranded mRNA Sample Prep Kit

474 with oligo(dT) selection (Illumina). 125-cycle paired-end sequencing was performed on an

475 Illumina HiSeq 2500 instrument (12 libraries/lane). An average of 23.4 million reads was 476 generated for each sample. 
ROR2 multiple sequence alignment

479 Amino acid sequences for vertebrate ROR2 and invertebrate ROR homologs were downloaded

480 from Ensembl (ensembl.org) or NCBI (ncbi.nlm.nih.gov/gene). Clustal Omega multiple

481 sequence alignments were performed and visualized with the R package msa v1.18.0 ${ }^{60}$.

482

483 Whole-mount RNA in situ hybridization (ISH)

484 ISH probe templates were generated by PCR amplification of a portion of pigeon ROR2 (692 bp 485 amplicon) or WNT5A (783 bp amplicon) from a pooled cDNA library generated from $\mathrm{HH} 21$, $486 \mathrm{HH} 25$, and HH29 Racing Homer embryos using the following primer sets: ROR2-forward: 5'487 GGAACCGACAGGTTCTACCA-3', ROR2-reverse: 5'-TGCTTCGTCCATCTGAAGTG-3', 488 WNT5A-forward: 5'-CATAGTGGCTCTGGCCATTT-3', $\quad$ WNT5A-reverse: $\quad$ 5'489 CCCCGACTGTTGAGTTTCAT-3'. ROR2 and WNT5A amplicons were cloned into pGEM-T 490 Easy (Promega) and confirmed by Sanger sequencing. Antisense and sense RNA probes were 491 generated by in vitro transcription as previously described ${ }^{61}$. For ROR2, pGEM-ROR2 was 492 digested with Ncol or Sall and transcribed with SP6 or T7 RNA polymerase, respectively. For 493 WNT5A, pGEM-WNT5A was digested with Kpn1 or Nco1 and transcribed with T7 or SP6 RNA 494 polymerase, respectively.

495 Racing Homer embryos used for ISH were dissected from eggs at the desired embryonic 496 stage and fixed overnight in $4 \%$ paraformaldehyde at $4^{\circ} \mathrm{C}$ on a shaking table. Embryos were 497 subsequently dehydrated into $100 \% \mathrm{MeOH}$ and stored at $-20^{\circ} \mathrm{C}$. Whole-mount ISH was 498 performed following a protocol optimized for avian embryos 499 (geisha.arizona.edu/geisha/protocols.jsp). For each experiment, antisense or sense probes 500 were applied to stage-matched embryos.

501

\section{Quantification and Statistical Analysis}

503 Linear measurement analysis 
504 For each $F_{2}$ individual and the cross founders, beak and braincase length were determined by

505 calculating the linear distance between landmark pairs (beak: landmarks 1 and 2; braincase:

506 landmarks 1 and $3,{ }^{24}$ ) using the interlmkdist function from the R package geomorph v3.3.1 ${ }^{62-64}$.

507 Raw beak length measurements were fit to a linear regression model (beak length body

508 mass) and residuals were calculated in $\mathrm{R}$ v3.6.3 ${ }^{65}$.

509

510 Geometric morphometrics

511 Geometric morphometric analyses were performed in geomorph as described ${ }^{24}$. The NTsys

512 landmark point dataset was imported with the readland.nts function. Missing landmarks were

513 estimated using the function estimate. missing(method = "TPS"). Bilateral symmetry analysis

514 was performed via the bilat.symmetry(iter $=1$ ) function and the symmetrical component of

515 shape variation was extracted. A Generalized Procrustes Analysis was performed using the

516 gpagen function. To analyze allometry, a linear model (shape centroid size) was fit using the

517 procD.Im function and residuals were used for analysis of allometry-free shape. Principal

518 components analysis was performed using the gm.prcomp function. Integration of beak and

519 braincase shape was analyzed using the two.b.pls function.

520 Shape changes were visualized with geomorph and the R package Morpho v2.8

521 (https://github.com/zarquon42b/Morpho). The geomorph function plotRefToTarget was used to

522 generate wireframes. Surface mesh deformations, heatmaps, and movies were generated in

523 Morpho with the tps3d, shade3d, meshDist, and warpmovie3d functions. For all mesh-based

524 visualizations, deformations were applied to a reference mesh, which was generated by warping

525 a $\mathrm{RH} \times \mathrm{OGO} \mathrm{F}_{2}$ mesh to the mean shape.

526

527 QTL mapping

528 QTL mapping was performed using the R package R/qtl v1.46-2 ${ }^{66}$. Single-QTL genome scans 529 were performed using the scanone function with Haley-Knott regression and sex as a covariate. 
530 The $5 \%$ genome-wide significance threshold was calculated by running scanone with 1000

531 permutation replicates. For each QTL, the 1.5-LOD support interval was calculated with the

532 lodint function, percent variance explained (PVE) was calculated with the fitqt/ function, and QTL

533 effects were estimated via the plotPXG function. We compared phenotypic means in $\mathrm{RH} \times \mathrm{OGO}$

$534 F_{2}$ genotypic groups at peak markers via one-way ANOVA and Tukey Test for pairwise

535 comparisons in R. Genes within QTL intervals were identified using a custom R script and

536 visualized using the $R$ packages ggplot2 v3.3.0 ${ }^{54}$ and gggenes $v 0.4 .0$

537 (https://github.com/wilkox/gggenes).

538

$539 \quad$ Variant calling and comparative genomic analyses

540 Variant calling was performed with FastQForward ${ }^{67}$, which wraps the BWA short read aligner ${ }^{68}$

541 and Sentieon (sentieon.com) variant calling tools to generate aligned BAM files (fastq2bam) and

542 variant calls in VCF format (bam2gvcf). Sentieon is a commercialized GATK equivalent pipeline

543 that allows users to follow GATK best practices using the Sentieon version of each tool

544 (broadinstitute.org/gatk/guide/best-practices and

545 support.sentieon.com/manual/DNAseq usage/dnaseq/). FastQForward manages distribution of

546 the workload to these tools on a compute cluster to allow for faster data-processing than when

547 calling these tools directly, resulting in runtimes as low as a few minutes per sample. Raw

548 sequencing reads from 54 newly resequenced individuals (described in Whole-genome

549 resequencing section) were aligned to the Cliv_2.1 reference assembly ${ }^{56}$ using fastq2bam.

550 Variant calling was performed for each newly resequenced individual, as well as 132 previously

551 resequenced individuals $27,30,50,69$, using bam2gvef and individual genome variant call format

552 (gVCF) files were created. Joint variant calling was performed on a total of 186 individuals using

553 the Sentieon GVCFtyper algorithm. The resulting VCF file was used for all subsequent genomic 554 analyses. 
Genome-wide Weir and Cockerham's $F_{S T}\left(w_{c} F_{S T}\right)$ and probabilistic $F_{S T}\left(p F_{S T}\right)$ were calculated using the GPAT++ toolkit within the VCFLIB software library (github.com/vcflib) as

557 previously described 27,30,50,69. Extended haplotype homozygosity (EHH) was calculated for

558 genomic scaffold ScoHet5_445.1 using the GPAT++ sequenceDiversity tool. Putatively 559 deleterious variants were identified using the Variant Annotation, Analysis, and Search Tool 560 (VAAST2, $\left.{ }^{36}\right)$, which was implemented as previously described ${ }^{27}$. For all comparative genomic 561 analyses, pigeons were binned into the following phenotypic groups:

562 Short beak (56 individuals, 31 breeds): African Owl, Australian Tumbler, Bacska

563 Tumbler, Berlin Short Face Tumbler, Budapest Tumbler, Canario Cropper, Catalonian Tumbler,

564 Chinese Nasal Tuft, Chinese Owl, Classic Old Frill, Damascene, Egyptian Swift, English Long

565 Face Tumbler, English Short Face Tumbler, Granadino Pouter, Hamburg Sticken, Helmet, 566 Italian Owl, Komorner Tumbler, Long Face Tumbler, Nun, Old German Owl, Oriental Frill,

567 Portuguese Tumbler, Rafeno Pouter, Russian Tumbler, Spanish Barb, Syrian Dewlap,

568 Taganrog Tumbler, Temeschburger Schecker, Uzbek Tumbler.

569 Medium or long beak (121 individuals, 58 breeds and feral): American Highflier, 570 American Show Racer, Archangel, Armenian Tumbler, Berlin Long Face Tumbler, Birmingham 571 Roller, Bokhara Trumpeter, Brunner Pouter, Carneau, Crested Saxon Field Color Pigeon, 572 Cumulet, Danish Tumbler, Danzig Highflier, Dragoon, English Carrier, English Magpie, English 573 Pouter, English Trumpeter, Fairy Swallow, Fantail, Feral, Franconian Trumpeter, Frillback, 574 German Beauty, Hungarian Giant House Pigeon, Ice Pigeon, Indian Fantail, Iranian Tumbler, 575 Jacobin, King, Lahore, Laugher, Lebanon, Marchenero Pouter, Mindian Fantail, Miniature 576 American Crest, Modena, Mookee, Naked Neck, Norwich Cropper, Old Dutch Capuchin, 577 Oriental Roller, Parlor Roller, Polish Lynx, Pomeranian Pouter, Pygmy Pouter, Racing Homer, 578 Rhine Ringbeater, Runt, Saint, Saxon Monk, Saxon Pouter, Scandaroon, Schalkaldener 579 Mohrenkopf, Shakhsharli, Starling, Turkish Tumbler, Vienna Medium Face Tumbler, West of 580 England. 
582 RNA-seq analysis

583 Analysis of RNA-seq data was performed as previously described ${ }^{70}$. Briefly, sequencing read

584 quality was assessed with FastQC (Babraham Bioinformatics). Illumina adapters were trimmed

585 and reads were aligned to the pigeon Cliv_2.1 reference assembly (Holt et al., 2018) using

586 STAR v2.5.0a ${ }^{71}$ using the 2-pass mode. GTF annotation files were used to guide spliced read

587 alignments. Mapped reads were assigned to genes using featureCounts from the Subread

588 package version 1.5.1. Transcript abundance (TPM) was quantified using Salmon v1.3.0 ${ }^{72}$.

589 Differential expression analyses were performed with the R package DESeq2 version 1.12.4

590 (Love et al., 2014).

591 


\section{References}

594 1. Twigg, S.R.F., and Wilkie, A.O.M. (2015). New insights into craniofacial malformations. Hum. Mol. Genet. 24, R50-R59.

2. Fish, J.L. (2016). Developmental mechanisms underlying variation in craniofacial disease and evolution. Developmental Biology 415, 188-197.

3. Christie, W., and Wriedt, C. (1924). Die Vererbung von Zeichnungen, Farben und anderen Charakteren bei Tauben. Z.Ver-erbungslehre 32, 233-298.

4. Hollander, W.F. (1983). Origins and Excursions in Pigeon Genetics: A Compilation (The Ink Spot).

5. Sell, A. (2012). Pigeon Genetics: Applied Genetics in the Domestic Pigeon (Sell Publishing).

6. Topczewski, J., Dale, R.M., and Sisson, B.E. (2011). Planar cell polarity signaling in craniofacial development. Organogenesis 7, 255-259.

7. Mayor, R., and Theveneau, E. (2014). The role of the non-canonical Wnt-planar cell polarity pathway in neural crest migration. Biochem J 457, 19-26.

8. Afzal, A.R., Rajab, A., Fenske, C.D., Oldridge, M., Elanko, N., Ternes-Pereira, E., Tüysüz, B., Murday, V.A., Patton, M.A., Wilkie, A.O.M., et al. (2000). Recessive Robinow syndrome, allelic to dominant brachydactyly type B, is caused by mutation of ROR2. Nat Genet 25, 419-422.

9. van Bokhoven, H., Celli, J., Kayserili, H., van Beusekom, E., Balci, S., Brussel, W., Skovby, F., Kerr, B., Percin, E.F., Akarsu, N., et al. (2000). Mutation of the gene encoding the ROR2 tyrosine kinase causes autosomal recessive Robinow syndrome. Nat Genet 25, 423-426.

10. Tokita, M., Yano, W., James, H.F., and Abzhanov, A. (2017). Cranial shape evolution in adaptive radiations of birds: comparative morphometrics of Darwin's finches and Hawaiian honeycreepers. Phil. Trans. R. Soc. B 372, 20150481.

11. Navalón, G., Marugán-Lobón, J., Bright, J.A., Cooney, C.R., and Rayfield, E.J. (2020). The consequences of craniofacial integration for the adaptive radiations of Darwin's finches and Hawaiian honeycreepers. Nat Ecol Evol 4, 270-278.

12. Bosse, M., Spurgin, L.G., Laine, V.N., Cole, E.F., Firth, J.A., Gienapp, P., Gosler, A.G., McMahon, K., Poissant, J., Verhagen, I., et al. (2017). Recent natural selection causes adaptive evolution of an avian polygenic trait. Science 358, 365-368.

13. vonHoldt, B.M., Kartzinel, R.Y., Huber, C.D., Le Underwood, V., Zhen, Y., Ruegg, K., Lohmueller, K.E., and Smith, T.B. (2018). Growth factor gene IGF1 is associated with bill size in the black-bellied seedcracker Pyrenestes ostrinus. Nat Commun 9, 4855.

14. Abzhanov, A. (2004). Bmp4 and Morphological Variation of Beaks in Darwin's Finches. Science 305, 1462-1465. 
15. Abzhanov, A., Kuo, W.P., Hartmann, C., Grant, B.R., Grant, P.R., and Tabin, C.J. (2006). The calmodulin pathway and evolution of elongated beak morphology in Darwin's finches. $442,5$.

16. Mallarino, R., Grant, P.R., Grant, B.R., Herrel, A., Kuo, W.P., and Abzhanov, A. (2011). Two developmental modules establish 3D beak-shape variation in Darwin's finches. Proceedings of the National Academy of Sciences 108, 4057-4062.

17. Lamichhaney, S., Berglund, J., Almén, M.S., Maqbool, K., Grabherr, M., Martinez-Barrio, A., Promerová, M., Rubin, C.-J., Wang, C., Zamani, N., et al. (2015). Evolution of Darwin's finches and their beaks revealed by genome sequencing. Nature 518, 371-375.

18. Lamichhaney, S., Han, F., Berglund, J., Wang, C., Almen, M.S., Webster, M.T., Grant, B.R., Grant, P.R., and Andersson, L. (2016). A beak size locus in Darwins finches facilitated character displacement during a drought. Science 352, 470-474.

19. Baptista, L.F., Gómez, J.E.M., and Horblit, H.M. (2009). DARWIN'S PIGEONS AND THE EVOLUTION OF THE COLUMBIFORMS: RECAPITULATION OF ANCIENT GENES. 23.

20. Young, N.M., Linde-Medina, M., Fondon, J.W., Hallgrímsson, B., and Marcucio, R.S. (2017). Craniofacial diversification in the domestic pigeon and the evolution of the avian skull. Nat Ecol Evol 1, 0095.

21. Des Moore (2018). Judging Racing Pigeons. Des Moore's Pigeon Domain. http://desmoore.tripod.com/id56.html.

22. Old German Owl Club Standard (2019). Old German Owl Club. http://ogoc.org/standard.htm.

23. Hallgrímsson, B., Katz, D.C., Aponte, J.D., Larson, J.R., Devine, J., Gonzalez, P.N., Young, N.M., Roseman, C.C., and Marcucio, R.S. (2019). Integration and the Developmental

24. Boer, E. F., Maclary, E. T., and Shapiro, M. D. (2021). Complex genetic architecture of Genetics of Allometry. Integrative and Comparative Biology 59, 1369-1381. three-dimensional craniofacial shape variation in domestic pigeons.

25. Wexelsen, H. (1937). Size inheritance in pigeons. Journal of Experimental Zoology 76, 161186.

26. Stringham, S.A., Mulroy, E.E., Xing, J., Record, D., Guernsey, M.W., Aldenhoven, J.T., Osborne, E.J., and Shapiro, M.D. (2012). Divergence, convergence, and the ancestry of feral populations in the domestic rock pigeon. Curr Biol 22, 302-8.

27. Shapiro, M.D., Kronenberg, Z., Li, C., Domyan, E.T., Pan, H., Campbell, M., Tan, H., Huff, C.D., Hu, H., Vickrey, A.l., et al. (2013). Genomic diversity and evolution of the head crest in the rock pigeon. Science 339, 1063-7.

28. Pacheco, G., van Grouw, H., Shapiro, M.D., Gilbert, M.T.P., and Vieira, F.G. (2020). Darwin's Fancy Revised: An Updated Understanding of the Genomic Constitution of Pigeon Breeds. Genome Biology and Evolution 12, 136-150. 
665

666

667

668

669

670

671

672

673

674

675

676

677

678

679

680

681

682

683

684

685

686

687

688

689

690

691

692

693

694

695

696

697

698

699

700

701

29. Weir, B.S., and Cockerham, C.C. (1984). Estimating F-Statistics for the Analysis of Population Structure. Evolution 38, 1358.

30. Domyan, E.T., Kronenberg, Z., Infante, C.R., Vickrey, A.I., Stringham, S.A., Bruders, R., Guernsey, M.W., Park, S., Payne, J., Beckstead, R.B., et al. (2016). Molecular shifts in limb identity underlie development of feathered feet in two domestic avian species. Elife 5, e12115.

31. Stricker, S., Rauschenberger, V., and Schambony, A. (2017). ROR-Family Receptor Tyrosine Kinases. In Current Topics in Developmental Biology (Elsevier), pp. 105-142.

32. DeChiara, T.M., Kimble, R.B., Poueymirou, W.T., Rojas, J., Masiakowski, P., Valenzuela, D.M., and Yancopoulos, G.D. (2000). Ror2, encoding a receptor-like tyrosine kinase, is required for cartilage and growth plate development. Nat Genet 24, 271-274.

33. Schwabe, G.C., Trepczik, B., Süring, K., Brieske, N., Tucker, A.S., Sharpe, P.T., Minami, Y., and Mundlos, S. (2004). Ror2 knockout mouse as a model for the developmental pathology of autosomal recessive Robinow syndrome: Developmental Model for Robinow Syndrome. Dev. Dyn. 229, 400-410.

34. Raz, R., Stricker, S., Gazzerro, E., Clor, J.L., Witte, F., Nistala, H., Zabski, S., Pereira, R.C., Stadmeyer, L., Wang, X., et al. (2008). The mutation ROR2W749X, linked to human BDB, is a recessive mutation in the mouse, causing brachydactyly, mediating patterning of joints and modeling recessive Robinow syndrome. Development 135, 1713-1723.

35. Mosca, F., and Ipsom, J.P. (2005). Standard of the Chinese Nasal Tuft. https://www.angelfire.com/ga3/pigeongenetics/nasaltuftstandard.html/.

36. Hu, H., Huff, C.D., Moore, B., Flygare, S., Reese, M.G., and Yandell, M. (2013). VAAST 2.0: improved variant classification and disease-gene identification using a conservationcontrolled amino acid substitution matrix. Genet Epidemiol 37, 622-634.

37. Chen, Y., Bellamy, W.P., Seabra, M.C., Field, M.C., and Ali, B.R. (2005). ER-associated protein degradation is a common mechanism underpinning numerous monogenic diseases including Robinow syndrome. Human Molecular Genetics 14, 2559-2569.

38. Oishi, I., Suzuki, H., Onishi, N., Takada, R., Kani, S., Ohkawara, B., Koshida, I., Suzuki, K., Yamada, G., Schwabe, G.C., et al. (2003). The receptor tyrosine kinase Ror2 is involved in non-canonical Wnt5a/JNK signalling pathway: Role of Ror2 in Wnt5a signalling pathway. Genes to Cells 8,645-654.

39. Li, Y., Han, X., Xu, W., Rao, Z., and Li, X. (2019). Purification and characterization of the extracellular region of human receptor tyrosine kinase like orphan receptor 2 (ROR2). Protein Expression and Purification 158, 74-80.

40. Ali, B.R., Jeffery, S., Patel, N., Tinworth, L.E., Meguid, N., Patton, M.A., and Afzal, A.R. (2007). Novel Robinow syndrome causing mutations in the proximal region of the frizzledlike domain of ROR2 are retained in the endoplasmic reticulum. Hum Genet 122, 389-395. 
702

703

704

705

706

707

708

709

710

711

712

713

714

715

716

717

718

719

720

721

722

723

724

725

726

727

728

729

730

731

732

733

734

735

736

737

738

739

740

41. Matsuda, T., Nomi, M., Ikeya, M., Kani, S., Oishi, I., Terashima, T., Takada, S., and Minami, Y. (2001). Expression of the receptor tyrosine kinase genes, Ror1 and Ror2, during mouse development. Mechanisms of Development 105, 153-156.

42. Stricker, S., Verhey Van Wijk, N., Witte, F., Brieske, N., Seidel, K., and Mundlos, S. (2006). Cloning and expression pattern of chicken Ror2 and functional characterization of truncating mutations in Brachydactyly type B and Robinow syndrome. Dev. Dyn. 235, 3456-3465.

43. Hamburger, V., and Hamilton, H.L. (1951). A series of normal stages in the development of the chick embryo. J. Morphol. 88, 49-92.

44. Smith, F.J., Percival, C.J., Young, N.M., Hu, D., Schneider, R.A., Marcucio, R.S., and Hallgrimsson, B. (2015). Divergence of craniofacial developmental trajectories among avian embryos: Craniofacial Trajectories Among Avian Embryos. Dev. Dyn. 244, 1158-1167.

45. Geetha-Loganathan, P., Nimmagadda, S., Antoni, L., Fu, K., Whiting, C.J., Francis-West, P., and Richman, J.M. (2009). Expression of WNT signalling pathway genes during chicken craniofacial development. Dev. Dyn. 238, 1150-1165.

46. Bult, C.J., Blake, J.A., Smith, C.L., Kadin, J.A., Richardson, J.E., and Mouse Genome Database Group (2019). Mouse Genome Database (MGD) 2019. Nucleic Acids Res 47, D801-D806.

47. Vickrey, A.I., Domyan, E.T., Horvath, M.P., and Shapiro, M.D. (2015). Convergent Evolution of Head Crests in Two Domesticated Columbids Is Associated with Different Missense Mutations in EphB2. Mol Biol Evol 32, 2657-2664.

48. Li, J., Lee, M., Davis, B.W., Lamichhaney, S., Dorshorst, B.J., Siegel, P.B., and Andersson*, L. (2020). Mutations Upstream of the TBX5 and PITX1 Transcription Factor Genes Are Associated with Feathered Legs in the Domestic Chicken. Molecular Biology and Evolution $37,2477-2486$.

49. Poelstra, J.W., Vijay, N., Hoeppner, M.P., and Wolf, J.B.W. (2015). Transcriptomics of colour patterning and coloration shifts in crows. Mol Ecol 24, 4617-4628.

50. Vickrey, A.I., Bruders, R., Kronenberg, Z., Mackey, E., Bohlender, R.J., Maclary, E.T., Maynez, R., Osborne, E.J., Johnson, K.P., Huff, C.D., et al. (2018). Introgression of regulatory alleles and a missense coding mutation drive plumage pattern diversity in the rock pigeon. Elife 7 .

51. Knief, U., Bossu, C.M., Saino, N., Hansson, B., Poelstra, J., Vijay, N., Weissensteiner, M., and Wolf, J.B.W. (2019). Epistatic mutations under divergent selection govern phenotypic variation in the crow hybrid zone. Nat Ecol Evol 3, 570-576.

52. Guernsey, M.W., Ritscher, L., Miller, M.A., Smith, D.A., Schöneberg, T., and Shapiro, M.D. (2013). A Val85Met mutation in melanocortin-1 receptor is associated with reductions in eumelanic pigmentation and cell surface expression in domestic rock pigeons (Columba livia). PLoS One 8, e74475.

53. Domyan, E.T., Guernsey, M.W., Kronenberg, Z., Krishnan, S., Boissy, R.E., Vickrey, A.I., Rodgers, C., Cassidy, P., Leachman, S.A., Fondon, J.W., 3rd, et al. (2014). Epistatic and 
combinatorial effects of pigmentary gene mutations in the domestic pigeon. Curr Biol 24, 459-64.

54. Wickham, H. (2016). ggplot2: elegant graphics for data analysis Second edition. (Springer).

744

745

746

747

748

749

750

751

752

753

754

755

756

757

758

759

760

761

762

763

764

765

766

767

768

769

770

771

772

773

774

775

55. Martin, M. (2011). Cutadapt removes adapter sequences from high-throughput sequencing reads. EMBnet j. 17, 10.

56. Holt, C., Campbell, M., Keays, D.A., Edelman, N., Kapusta, A., Maclary, E., E, T.D., Suh, A., Warren, W.C., Yandell, M., et al. (2018). Improved Genome Assembly and Annotation for the Rock Pigeon (Columba livia). G3 (Bethesda) 8, 1391-1398.

57. Langmead, B., and Salzberg, S.L. (2012). Fast gapped-read alignment with Bowtie 2. Nat Methods 9, 357-359.

58. Hamburger, V., and Hamilton, H.L. (1992). A series of normal stages in the development of the chick embryo. 1951. Dev Dyn 195, 231-72.

59. Fridolfsson, A.-K., and Ellegren, H. (1999). A Simple and Universal Method for Molecular Sexing of Non-Ratite Birds. Journal of Avian Biology 30,116.

60. Bodenhofer, U., Bonatesta, E., Horejš-Kainrath, C., and Hochreiter, S. (2015). msa: an R package for multiple sequence alignment. Bioinformatics, btv494.

61. Boer, E.F., Howell, E.D., Schilling, T.F., Jette, C.A., and Stewart, R.A. (2015). Fascin1Dependent Filopodia are Required for Directional Migration of a Subset of Neural Crest Cells. PLoS Genet 11, e1004946.

62. Adams, D.C., Collyer, M.L., and Kaliontzopoulou, A. (2020). Geomorph: Software for geometric morphometric analyses. R package version 3.2.1.

63. Collyer, M.L., and Adams, D.C. (2018). RRPP: An R package for fitting linear models to high-dimensional data using residual randomization. Methods Ecol Evol 9, 1772-1779.

64. Collyer, M.L., and Adams, D.C. (2020). RRPP: Linear Model Evaluation with Randomized Residuals in a Permutation Procedure, R package version 0.5.2.

65. R Core Team (2020). R: A Language and Environment for Statistical Computing (R Foundation for Statistical Computing).

66. Broman, K.W., Wu, H., Sen, S., and Churchill, G.A. (2003). R/qtl: QTL mapping in experimental crosses. Bioinformatics 19, 889-890.

67. Carson Holt FastQForward.

68. Li, H., and Durbin, R. (2009). Fast and accurate short read alignment with Burrows-Wheeler transform. Bioinformatics 25, 1754-1760.

69. Bruders, R., Van Hollebeke, H., Osborne, E.J., Kronenberg, Z., Maclary, E., Yandell, M., and Shapiro, M.D. (2020). A copy number variant is associated with a spectrum of pigmentation patterns in the rock pigeon (Columba livia). PLoS Genet 16, e1008274. 
70. Boer, E.F., Van Hollebeke, H.F., Park, S., Infante, C.R., Menke, D.B., and Shapiro, M.D. (2019). Pigeon foot feathering reveals conserved limb identity networks. Dev Biol 454, 128144.

71. Dobin, A., Davis, C.A., Schlesinger, F., Drenkow, J., Zaleski, C., Jha, S., Batut, P., Chaisson, M., and Gingeras, T.R. (2013). STAR: ultrafast universal RNA-seq aligner. Bioinformatics 29, 15-21.

72. Patro, R., Duggal, G., Love, M.I., Irizarry, R.A., and Kingsford, C. (2017). Salmon provides fast and bias-aware quantification of transcript expression. Nat Methods 14, 417-419. 

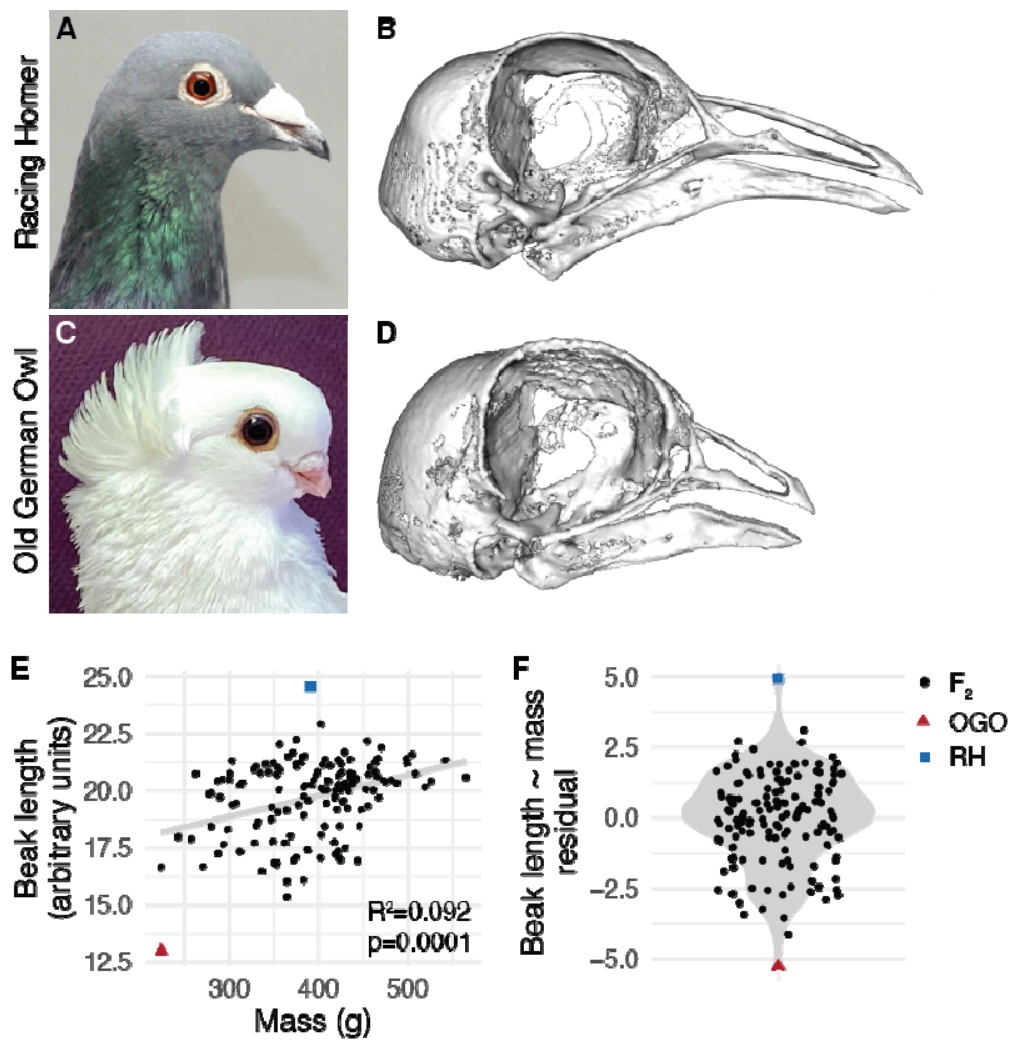

Figure 1. Beak length variation in a pigeon $\mathbf{F}_{2}$ intercross. (A) Representative image of the medium beak Racing Homer $(\mathrm{RH})$ breed (image credit: Sydney Stringham). (B) 3D surface model of the craniofacial skeleton of the male $\mathrm{RH}$ founder. (C) Representative image of the short beak Old German Owl (OGO) breed (image credit: Brian McCormick). (D) Surface model of the craniofacial skeleton of the female OGO founder. (E) Raw beak length (measured in arbitrary units) vs. total body mass (measured in grams) in the $\mathrm{RH} \times \mathrm{OGO}$ cross. Gray line indicates linear model from beak length $\sim$ mass regression. $(F)$ Distribution of residuals from beak length $\sim$ mass regression. For $(E-F)$, black dots denote $\mathrm{RH} \times \mathrm{OGO} \mathrm{F}_{2}$ individuals, red triangle is OGO founder, blue square is $\mathrm{RH}$ founder. 
A

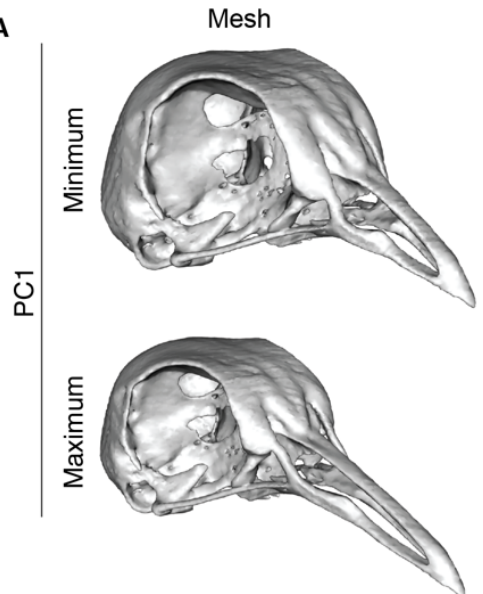

A

B

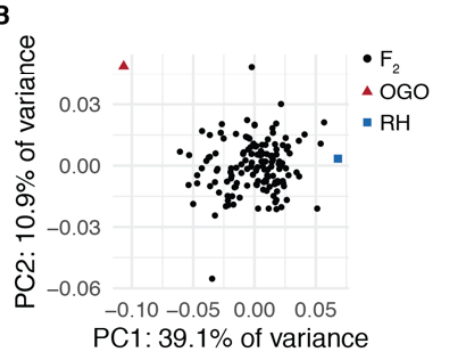

D

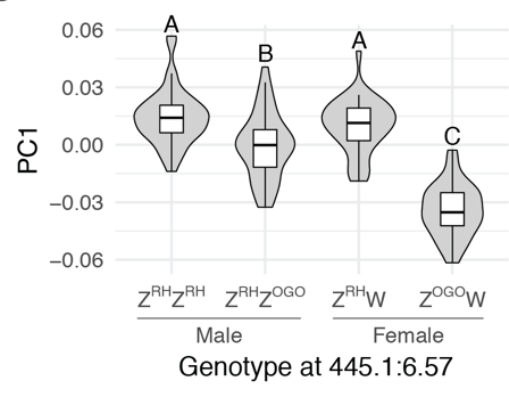

C
Wireframe
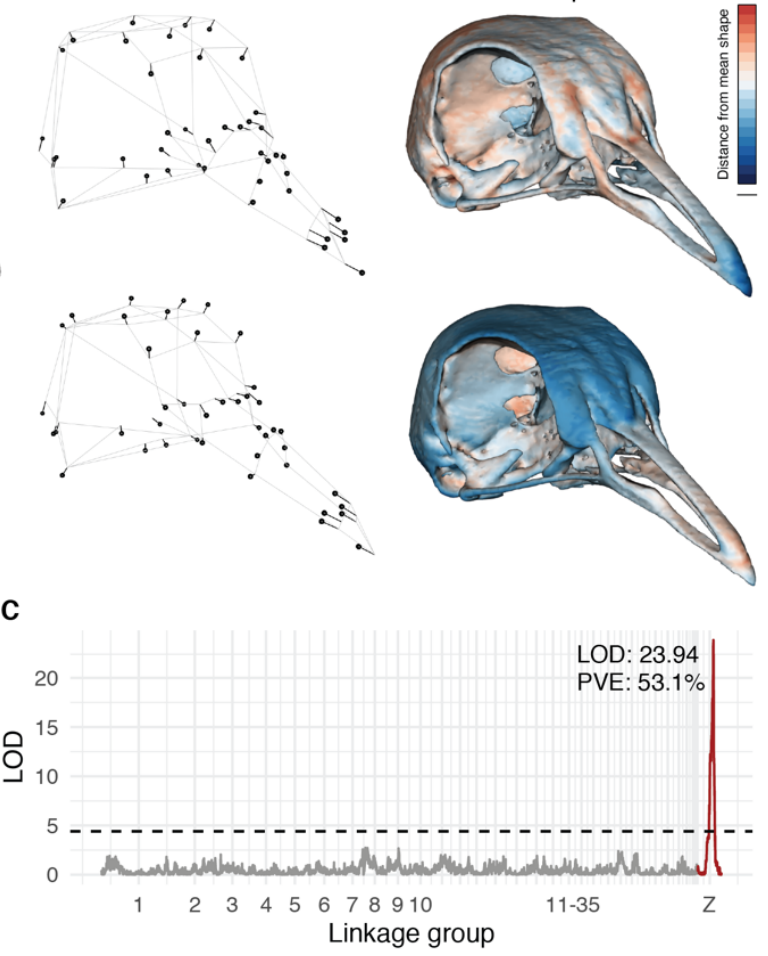

Figure 2. A major-effect QTL on the Z-chromosome is associated with principal component 1 (PC1) in the $R H \times O G O F_{2}$ population. (A) Visualizations of geometric morphometric PC1 minimum and maximum shapes three ways: warped 3D surface meshes (left), wireframes showing displacement of landmarks from mean shape (center), and heatmaps displaying regional shape variation (right). For warped meshes and wireframes, shape changes are magnified 1.5x to aid visualization. (B) Plot of PC1 vs. PC2. (C) Genome-wide QTL scan for PC1 reveals a significant QTL on the Z linkage group. (D) PC1 effect plot estimated from QTL peak marker. Letters denote significance groups; $\mathrm{RH}=$ allele from $\mathrm{RH}$ founder, $\mathrm{OGO}=$ allele from OGO founder. 
A

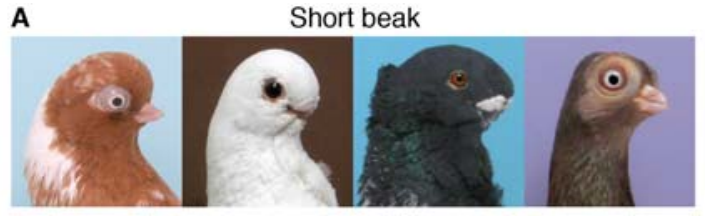

C $_{1.00}$

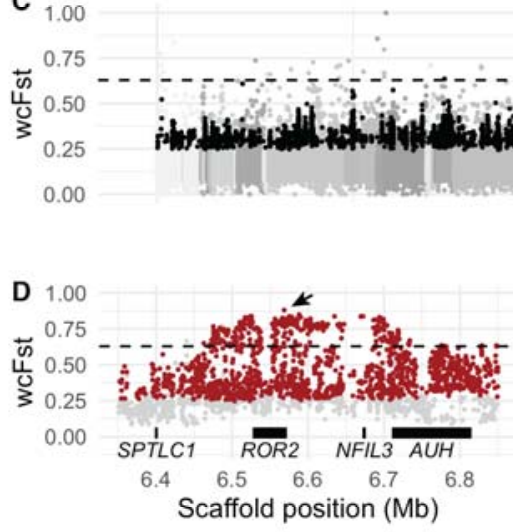

G
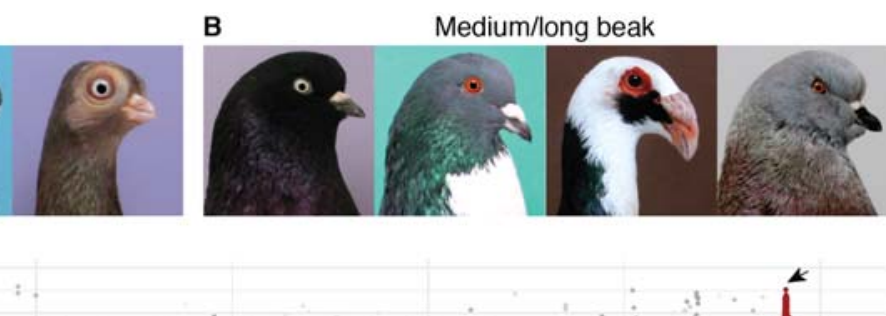

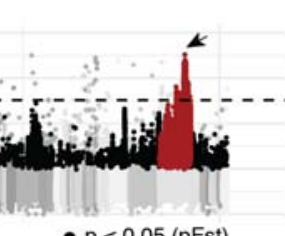

- $p<0.05$ (pFst) Genomic scaffolds

E $\quad 1.00$

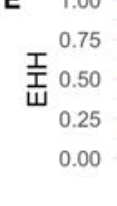

F

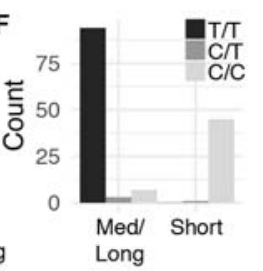

O Short

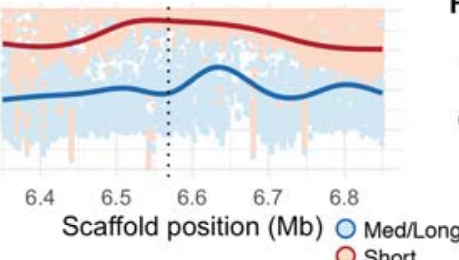

ROR2 kringle domain
808

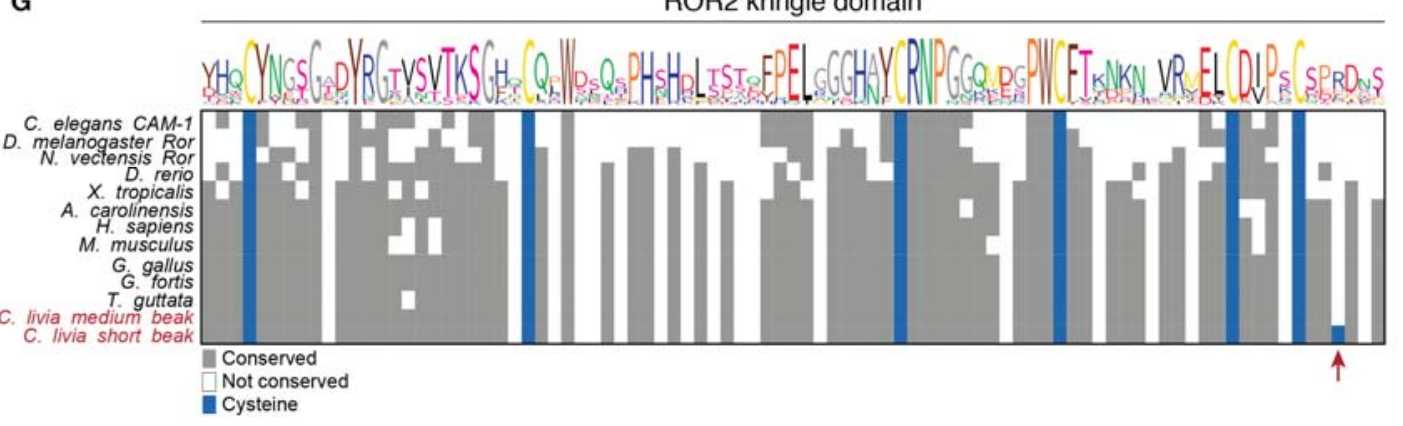

Figure 3. Comparison of short beak and medium/long beak pigeon genomes reveals ROR2 coding variant. (A-B) Representative images of individuals representing short beak (A) and medium or long beak (B) pigeon breeds (image credit: Thomas Hellmann). (A) Short beak pigeons, from left to right: English Short Face Tumbler, African Owl, Oriental Frill, Budapest Tumbler. (B) Medium/long beak pigeons, from left to right: West of England, Cauchois, Scandaroon, Show King. (C) Genome-wide scan for allele frequency differentiation between short beak $(n=56)$ and medium/long beak $(n=121)$ pigeons. (D) Region of peak allelic differentiation on ScoHet5_445.1; black horizontal bars represent four genes in the region. For (C-D), genomic scaffolds are colored in gray and ordered by genetic position in $\mathrm{RH} \times \mathrm{OGO}$ linkage map; black dots indicate SNPs that are significantly differentiated by $\mathrm{pF}_{\mathrm{ST}}$ (Bonferronicorrected p-value < 0.05); red dots are significant SNPs located on scaffold ScoHet5_445.1; dashed horizontal line represents threshold for genome-wide top $0.1 \%$ of differentiated SNPs by $\mathrm{wcF}_{\mathrm{ST}}$; arrow points to ScoHet5_445.1:6568443, the most differentiated SNP (wcF $\mathrm{ST}_{\mathrm{ST}} 0.88$ ) genome-wide. (E) Extended haplotype homozygosity in peak differentiated region; dotted vertical line indicates position of ScoHet5_445.1:6568443; smoothed lines represent local regression fitting ${ }^{54}(F)$ Histogram of genotypes at ScoHet5_445.1:6568443 in short beak and medium/long beak groups. $(G)$ Amino acid alignment of kringle domain from vertebrate ROR2 and invertebrate Ror homologs. The Ku2 allele causes an arginine-to-cysteine substitution in short beak pigeon breeds (indicated by red arrow). 

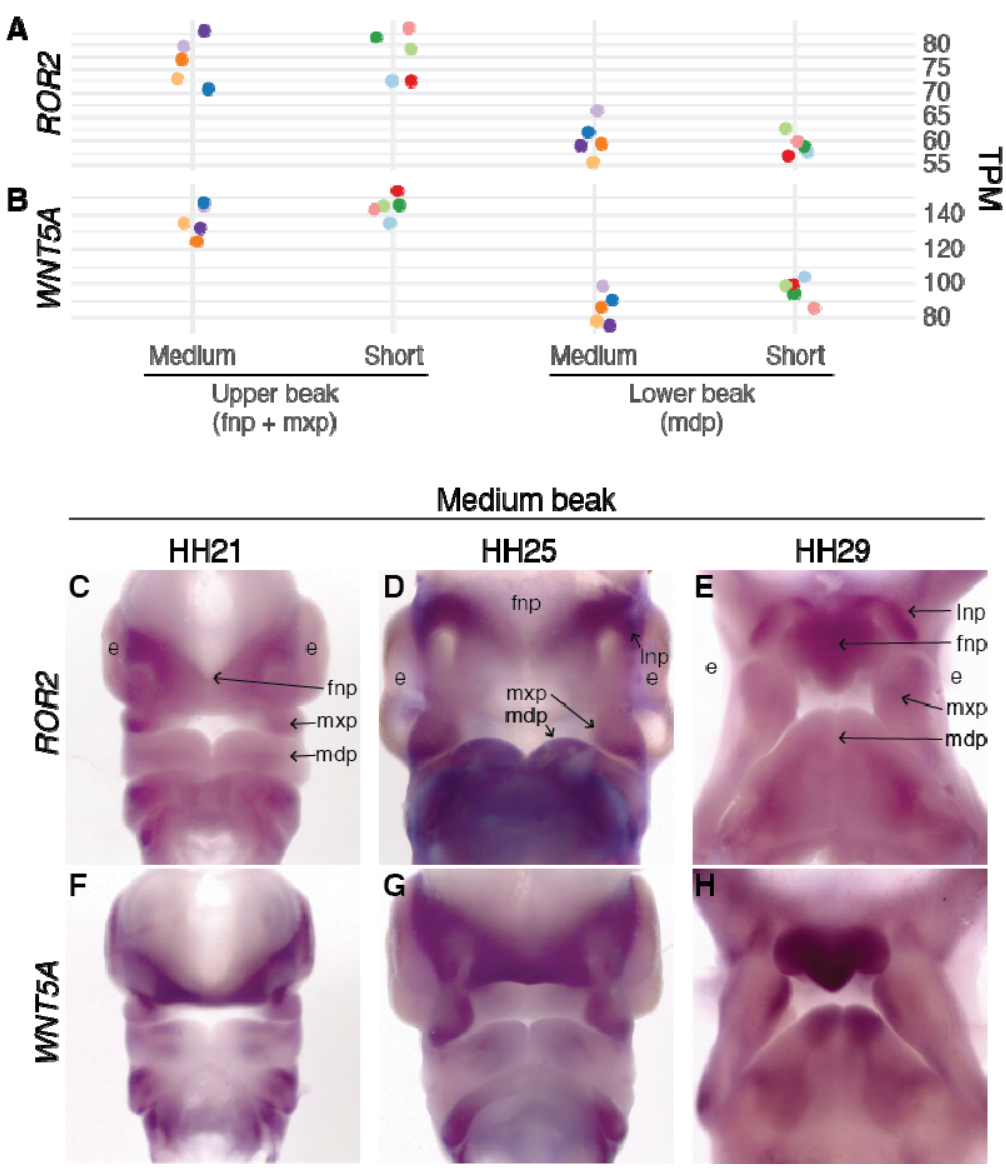

Figure 4. ROR2 and WNT5A expression in pigeon facial primordia. (A-B) ROR2 (A) and WNT5A (B) mRNA expression in facial primordia that will form the upper and lower beak from $\mathrm{HH} 29$ short and medium beak pigeon embryos. Each individual embryo is displayed in a different color. TPM, transcripts per million reads of mRNA-seq data. (C-H) Whole-mount in situ hybridization for ROR2 (C-E) and WNT5A (F-H) in medium beak pigeon embryos at $\mathrm{HH} 21$, $\mathrm{HH} 25$, and $\mathrm{HH} 29$. ROR2 is broadly expressed in facial primordia at all stages. WNT5A is strongly expressed in the frontonasal prominence and at the lateral edges of the maxillary and lateral nasal prominences, with increased expression at the edge of the mandibular prominence at $\mathrm{HH} 29$. Letters indicate embryonic tissues/structures: e=eye, fnp=frontonasal prominence, $\mathrm{l}=$ lateral nasal prominence, $\mathrm{mdp}=$ mandibular prominence, $\mathrm{mxp}=$ maxillary prominence. 


\section{Lateral}

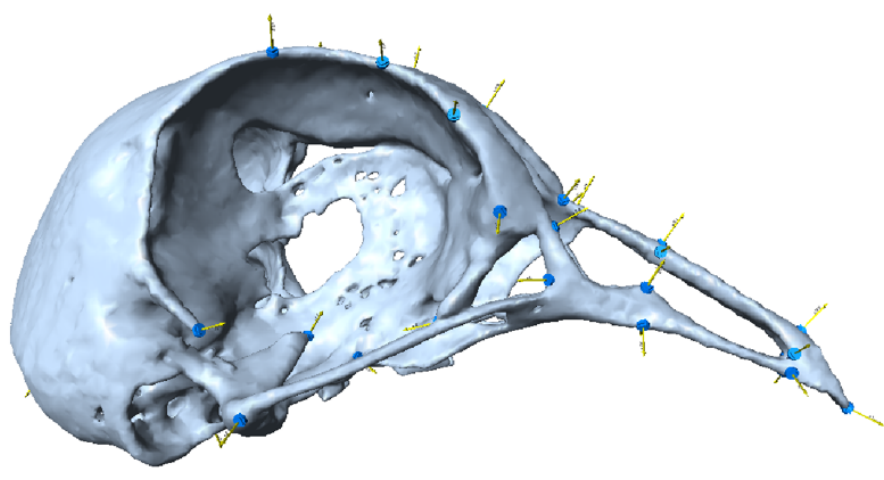

Dorsal

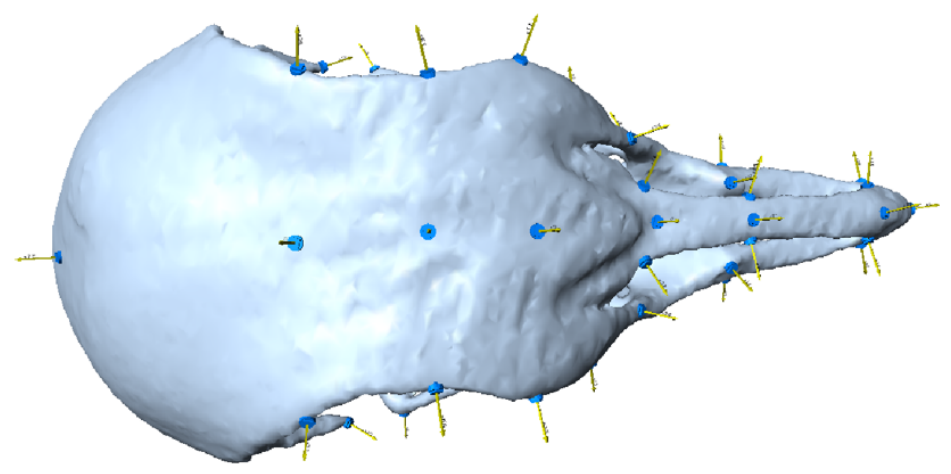

Ventral

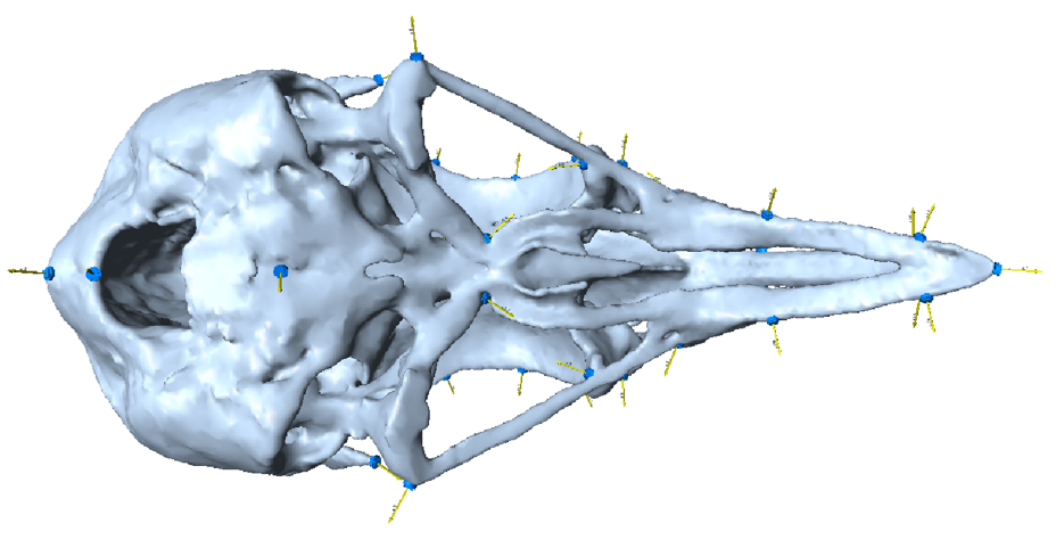

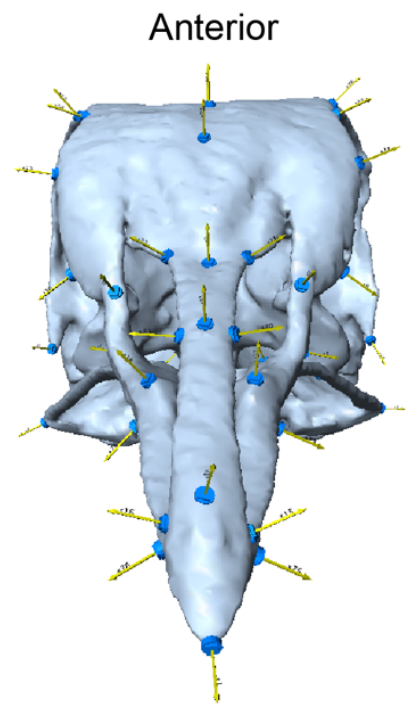

Posterior

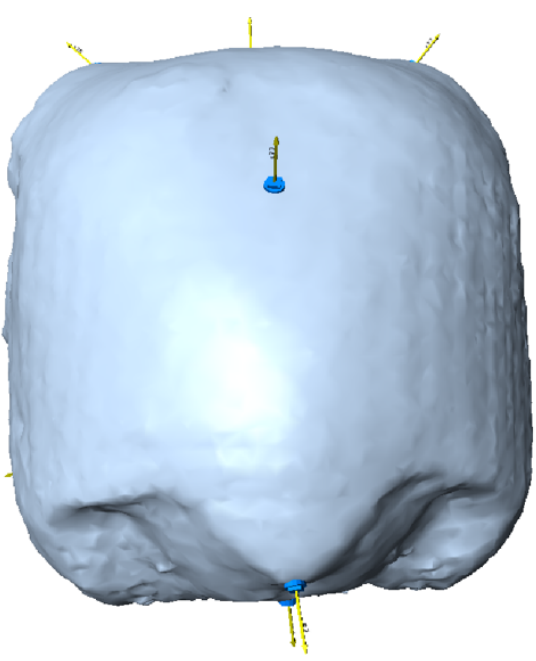

840

841

842

843

844
Supplemental Figure 1. Pigeon craniofacial landmark atlas. Landmarks are indicated by blue discs. 


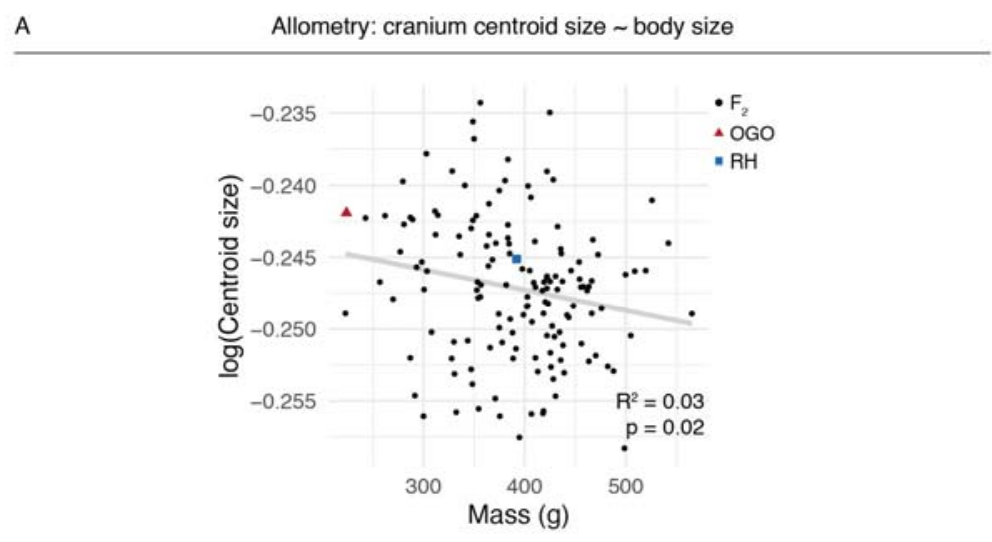

B Allometry: cranium shape $\sim$ cranium centroid size
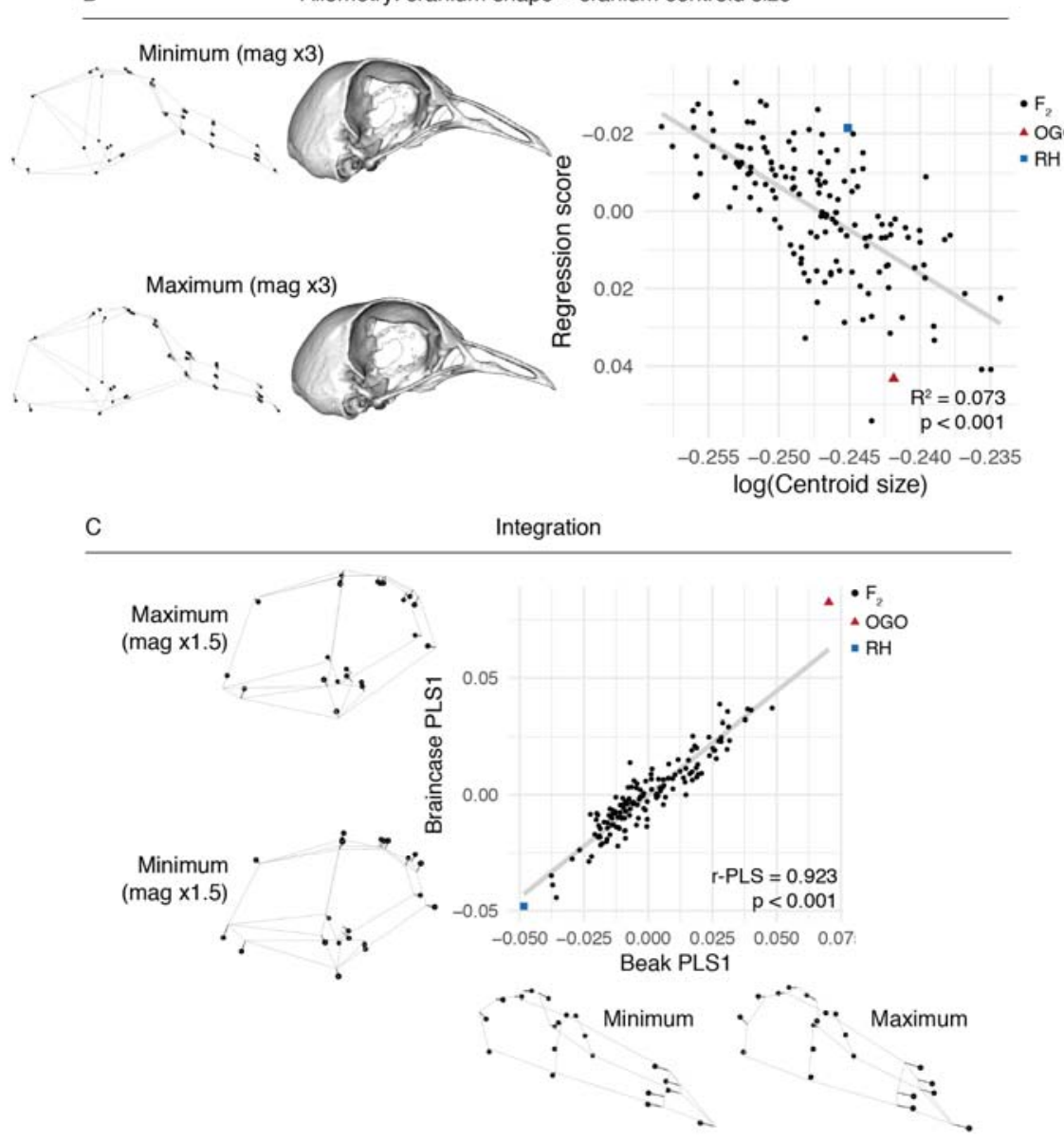

847 Supplemental Figure 2. Allometry and integration in the RH x OGO cross. (A) Cranium 848 centroid size body size (mass) linear regression. (B) Cranium shape centroid size linear regression. (B) Beak vs. braincase PLS1 shapes. Minimum and maximum shapes are depicted as wireframes and/or warped meshes along corresponding axis. 
A

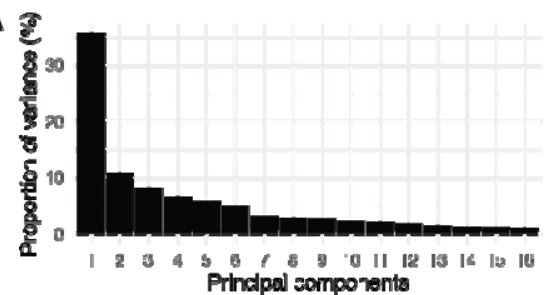

B $\quad 0.00$

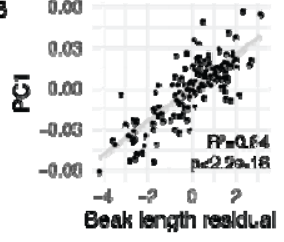

c

852 Supplemental Figure 3. Geometric morphometric analysis of craniofacial skeleton shape in RH x OGO cross. (A) Principal components (PCs) that together account for $90 \%$ of shape variation in craniofacial skeleton. (B) Scatter plot of beak length residual vs. PC1 score for all $\mathrm{RH} \times \mathrm{OGO} \mathrm{F}_{2}$ individuals. (C) Minimum and maximum PC2 shapes depicted as warped mesh (left), wireframe showing landmark displacement (center), or heatmap indicating regional shape changes (right). For mesh and wireframe models, shape change is magnified $1.5 x$ to aid visualization. 
A

Beak length residual

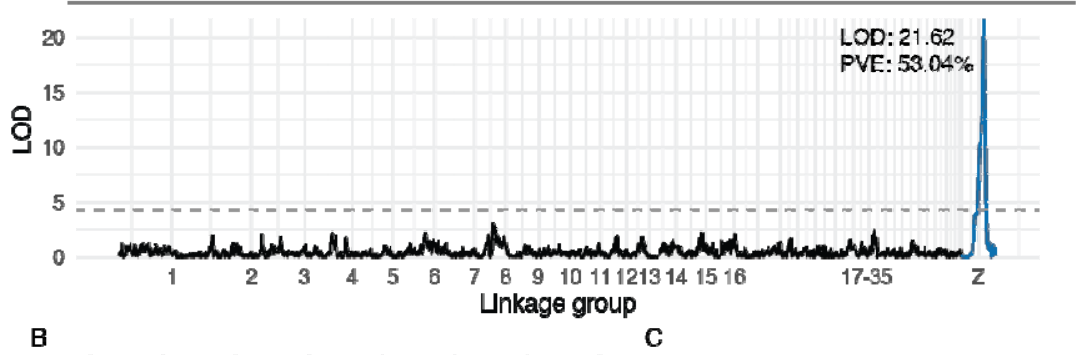

B

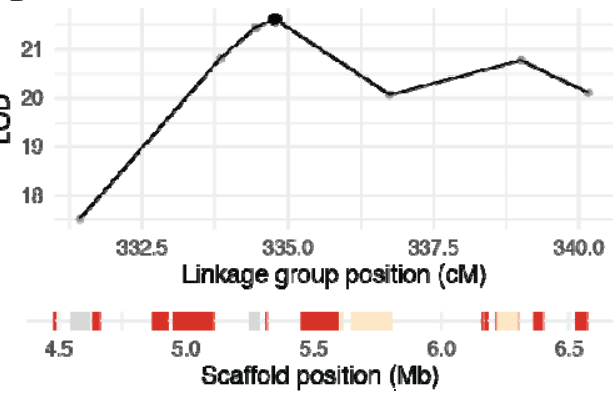

$\mathrm{C}$ for beak length using residuals from beak length $\sim$ body mass linear regression. (B) LOD support interval is nearly identical to PC1 QTL interval (displayed in Figure 2D). Genes in interval on ScoHet5_445.1 are displayed at bottom and color-coded by expression level. (C) Plot of QTL effects, using peak marker highlighted by black dot in (B). 
A

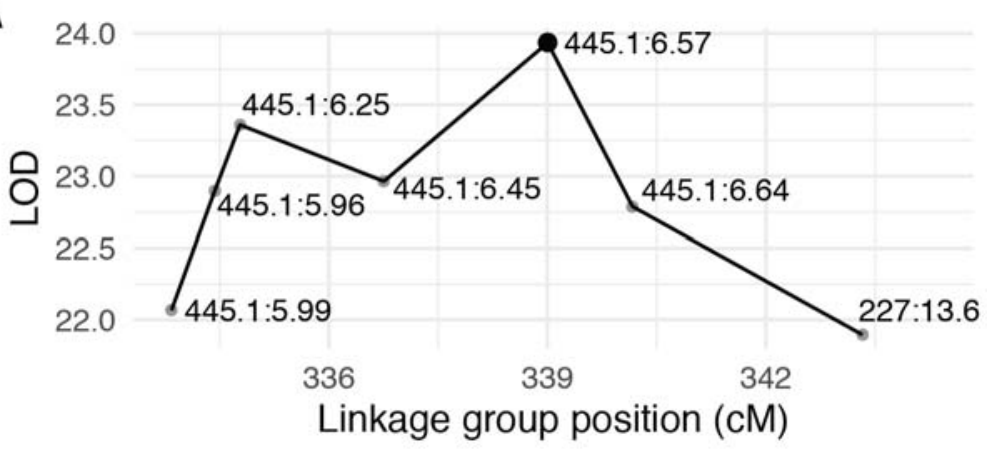

B

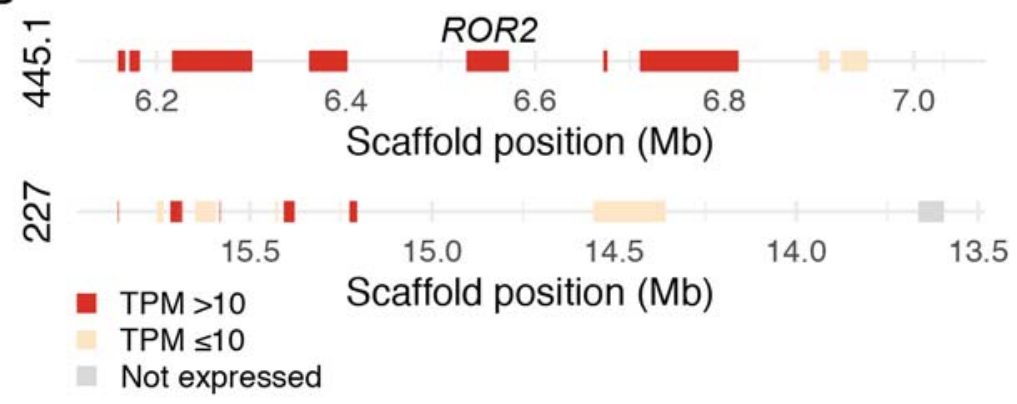

Supplemental Figure 5. PC1 QTL support interval. (A) LOD support interval for QTL on Z. Markers in interval are denoted with dots and labeled with genomic scaffold name (ScoHet5_445.1 or ScoHet5_227) and position (in Mb); black dot indicates QTL peak marker color-coded by mRNA expression level in facial primordia derived from $\mathrm{HH} 29 \mathrm{RH}$ embryos. $R O R 2$ is located directly under QTL peak. 

homozygosity (EHH) on scaffold ScoHet5_445.1 in short and medium/long beak pigeons. (A) $\mathrm{F}_{\mathrm{ST}}$ on ScoHet5_445.1. Boxed region indicates $\sim 293-\mathrm{kb}$ peak region displayed in Figure 3DE. (B) EHH on ScoHet5_445.1. Smoothed lines represent local regression fitting; dotted vertical line indicates position of ScoHet5_445.1:6568443.

Supplemental Movie 1. PC1 shape variation. Minimum to maximum, magnified 1.5x.

Supplemental Movie 2. PC2 shape variation. Minimum to maximum, magnified 1.5x. 